\title{
Efficient Mechanisms for Mergers and Acquisitions*
}

\author{
Sandro Brusco Giuseppe Lopomo David T. Robinson S. Viswanathan ${ }^{\dagger}$ \\ Running Head: Efficient Mechanisms for M\&A
}

\begin{abstract}
We characterize incentive-efficient merger outcomes when payments can be made both in cash and stock. Each firm has private information about both its stand-alone value and a component of the (possibly negative) potential synergies. We study two cases: when transfers can, and cannot, be made contingent on the value of any new firm. When they can, we show that redistributing shares of any non-merging firm generates information rents and provide necessary and sufficient conditions for the implementability of efficient merger rules. When they cannot, private information undermines efficiency more when it concerns stand-alone values than synergies. Here, acquisitions emerge as optimal mechanisms.
\end{abstract}

Keywords: Mergers and Acquisitions, Mechanism Design, Private Information

JEL: D82, G34

${ }^{*}$ First version: May 2005 — This revision: August 21, 2006.

†This paper supersedes a previous paper titled 'Merger Mechanisms' by Brusco, Lopomo and Viswanathan. We are grateful to two referees and the associate editor for many comments that helped us to improve the paper. We also gratefully acknowledge comments by Audra Boone, Tracy Lewis, Harold Mulherin, Jaideep Roy, participants in the Third International Conference on Game Theory in Mumbai, India, the Winter meetings of the Econometric Society, 2004, the conference on Auctions and Market Design, Milan 2003, and seminars at Rutgers University, Universitat Autònoma de Barcelona, Università Statale di Milano-Bicocca, Duke University and Università Cattolica di Milano. 


\section{Introduction}

Mergers and acquisitions are a ubiquitous feature of the modern corporate landscape. Indeed, in 2003, over a half-trillion dollars of merger activity occurred in the United States alone (Mergerstat Review, 2004). Invariably, each of these mergers involves firms acting on private information and subjective beliefs to structure a financial transaction that unites the assets of the two firms.

Given that asymmetric information problems are endemic to these types of transactions, when do efficient mergers occur? Are there too many mergers, or too few? How does the possibility of using stock, cash and other derivative securities affect the efficiency of the takeover process? Is the ownership structure of newly created partnerships affected by the initial information structure?

In this paper, we use a mechanism design approach to study these questions. The main advantage to this approach is its generality: in view of the Revelation Principle, any equilibrium of any conceivable game can be induced by the truth-telling equilibrium of an incentive-compatible revelation mechanism. Thus, our approach allows us to generalize beyond the specifics of any particular game form to derive the efficiency implications for all games with a common information structure. Given that equilibrium outcomes of bargaining games tend to be quite sensitive to small changes in the game form, the generality of our approach is especially valuable in the study of mergers, as evolving legal standards have effectively changed the permissible strategies of bidders and targets over time. ${ }^{1}$

The mechanism design approach is perhaps most commonly applied to the problem of a principal (e.g., monopolist) devising a menu of contracts to maximize profits from a group of potential agents with heterogeneous private valuations. In that setting, the mechanism design approach effectively ascribes total commitment power to a mechanism designer (the principal). Our analysis

\footnotetext{
${ }^{1}$ See Gilson and Black (1995) or Weston, Mitchell and Mulherin (2003) for discussions of the evolving legal standards surrounding the duties of a firm's management in the course of a takeover process.
} 
does not rely on the existence of a mechanism designer with total commitment power. Instead, we simply use the tools of mechanism design to identify the class of incentive compatible, individually rational actions that bidders and targets can take. We then examine the class of outcomes that these actions induce to ask whether efficient outcomes can be achieved, and if not, what second-best outcomes may look like.

We consider the case of a single target firm facing several potential suitors. Both the target and each of the $n$ potential acquirers have private information, not only about synergies they would bring to a merger, but also about one's own stand-alone value. In this setting, a merger mechanism consists of both a rule that identifies the winning bidder (if any), and a set of transfers among the $n+1$ parties. The set of transfers includes both cash and stock. Thus, the information structure of the problem we analyze is both general and realistic.

Our main goal is to identify the impact of private information on merger efficiency. The merger environment we study differs from the classic bi-lateral trade model of Myerson and Satterthwaite (1983) and its multilateral extensions studied by Makowski and Mezzetti (1993) and Williams (1999) in three key respects. First, in the case of mergers, firms can use shares as well as cash as a means of payment. Second, while most of the analysis in trading models focuses on the private values case, in the merger environment it is natural to assume that firms have interdependent values over the merged firm. Third, in the case of mergers, it is often reasonable to assume that transfers can be conditioned on ex post observables, while in many other cases this assumption may be less reasonable. We analyze both possibilities.

In principle, the possibility of using shares as a means of payment in addition to cash is a double-edged sword. On the one hand, stocks provide an additional degree of freedom that should make it easier to satisfy the incentive constraints imposed by the presence of private information. On the other hand, the true value of each firm's shares is privately known to its owner, hence share redistributions may well entail additional informational costs. 
Two key insights emerge from our analysis. First, contrary to what most of the previous literature suggests, private information about stand-alone values tends to be much more problematic than private information about potential synergies. Efficient mergers outcomes are always feasible when stand-alone values are known and there is private information only about synergies. Intuitively, this is because an increased willingness to merge by a bidder conveys good news about synergies, and this increases a potential partner's willingness to merge. On the other hand, since firms with higher stand-alone values are more reluctant to merge, eagerness to merge when standalone values are privately known can only convey bad news, and thus lower the interest of potential partners. The information rents that are then required to induce positive merger outcomes in this case hinder efficiency.

The second main insight involves the distinction between mergers and acquisitions. Whereas mergers involve shared ownership in the new firm, we define acquisitions to be the special case in which one party ends up owning $100 \%$ of the new firm, buying out the potential partner entirely. ${ }^{2}$ We show that acquisitions are optimal, in the sense of being best suited at achieving efficiency, because they minimize the frictions generated by private information. Thus our model delivers predictions about optimal ownership, even though we ignore moral hazard, making the allocation of control unimportant for realizing or producing synergies ex post.

To develop these insights, we study two polar situations. First, we assume that the value of any newly created firm can be verified ex post. This case is practically relevant, since contractual features of M\&A agreements like collars, contingent value rights or earn-outs allow a bidder to condition the offer on its ex post stock price. ${ }^{3}$

\footnotetext{
${ }^{2}$ Our manner of distinguishing mergers from acquisitions departs somewhat from the evolving legal definitions of mergers and acquisitions (see Gilson and Black (1995) and Weston, Mitchell, and Mulherin (2003)).

${ }^{3} \mathrm{~A}$ collar is a contractual feature in a stock-for-stock transaction in which the buyer agrees to reset the exchange ratio if its stock price drops below the level of the collar. Thus, this feature insulates the target firm against adverse
} 
In the case of perfect ex post verifiability, our main contribution consists in showing that the impact of private information on efficiency depends on the nature of the private information. Our analysis shows how to transform the general setting with interdependent values and multiple means of payment into a setting that is analogous to the private values trade model, so that the negative results of Myerson and Satterthwaite (1983) and Williams (1999) hold. First, we show that it is never optimal to transfer surplus among the firms not involved in the acquisition. Thus, the optimal mechanism never distributes stock or cash among losers, because doing so would generate needless information rents. On the other hand, the stock in the acquiring firm is equivalent to cash. Given these results, it is possible to devise sharing rules that transform our interdependent values problem into one of private values, in which classic impossibility results follow immediately. However, these results take on a different flavor in the context of mergers. In particular, we show that if the target perceives there to be a tiny chance that synergy values are so low that even the best merger would not be efficient, then the first-best merger outcome can never be implemented, even if this chance is vanishingly small. Thus, even though the value of any new firm can be verified ex post, the mere possibility of value destruction mergers forces any conceivable equilibrium in the merger market to leave some of the gains from merger unrealized. In addition, in a setting with multiple bidders, the second-best merger outcome almost always involves the target pairing with the bidder who does not offer the maximum synergies.

price changes in the bidder that occur between the announcement and the consummation of the merger. In contrast, Contingent Value Rights are portfolios of put options held by stockholders of the target firm that allow them to sell back their shares in the acquirer at fixed prices in the future. These put spreads insulate the target against price drops that occur after the merger is consummated. Both these mechanisms protect the target against uncertainty in the acquirer. Earn-outs protect the acquirer against uncertainty in the target. With earn-outs, buyers make supplementary payments to sellers after future earnings are realized. See Officer(2004) for collars, Hietala, Kaplan and Robinson (2003) for contingent value rights, or Datar, Frankel and Wolfson (2001) for the relation of adverse selection to earn-outs. 
These results illustrate that it is not necessary to resort to hubris, private benefits, entrenchment, or other agency costs to take a dim view of the efficiency of mergers. ${ }^{4}$ The sole presence of private information can be enough to prevent efficient merger outcomes, even when the value of any newly formed firm is verifiable ex post, and all actors are only seeking to maximize their total wealth. Private information can not only cause value-increasing mergers to go unexploited, but it can also cause firms to match with partners that offer smaller synergies than other potential partners.

The second polar case we consider is when the parties cannot contract on ex post outcomes. In particular, we assume that the value of any new firm is not verifiable. This case is reasonable in light of the recent theoretical and empirical work in the finance literature which highlights the fact that observed market prices may deviate from privately known stand-alone values, making it difficult to distinguish misreported stand-alone values from simple market corrections. ${ }^{5}$

In this case, our results are substantially different from the prior literature. First, in light of the inefficiencies identified above, it seems reasonable to conjecture that reducing the amount of verifiable information can only make matters worse for efficiency. But this is not generally so: the specific nature of private information plays a critical role in determining both whether efficient merger outcomes can be achieved, and what forms of the merger consideration minimize the deviation from the first-best.

When each firm's stand-alone value is privately known, and it is common knowledge that a merger is always welfare enhancing, the efficient merger rule can be implemented only if the gains from merging are sufficiently large. In contrast, when the private information concerns synergy

\footnotetext{
${ }^{4}$ Roll's (1986) hubris hypothesis of takeovers argues that hubris on the part of bidders causes them to ignore the market's assessment of the target's value and overpay by offering a premium over prevailing market prices.

${ }^{5}$ For theoretical work along these lines, see Rhodes-Kropf and Viswanathan (2005) or Shleifer and Vishny (2003); for empirical evidence, see Rhodes-Kropf, Robinson, and Viswanathan (2005).
} 
values, and the firms' stand-alone values are observable, it is always possible to design mechanisms implementing the efficient merger rule. In fact, this can be done by simply redistributing shares.

The information structure also plays a key role in determining who buys whom. Whenever there is more uncertainty about stand-alone values than synergies, the firm with the higher standalone values should own the firm ex post. Conversely, when synergies are more uncertain, the firm with the higher synergies should be the owner of the newly created firm.

Thus, when the merger consideration cannot be conditioned on ex post observable information, the nature of private information is crucial to the overall efficiency of takeovers. Intuitively, this is because eagerness to merge signals good news in situations where the uncertainty is about synergy values, and bad news when the uncertainty is about stand-alone values.

Comparing these two cases illustrates that both the possibility of achieving efficiency and the nature of incentive-efficient merger mechanisms is sensitive to the information structure. Mergers with shared ownership are optimal in the case where synergies are uncertain and stand-alone values are known. Acquisitions, in which one party buys out the other party entirely, are optimal in the case with privately known stand-alone values and known synergies.

Our findings contribute to the literature that examines mergers and acquisitions from a mechanism design perspective. Hansen (1985) first considered the case of multiple firms competing to acquire a target firm, and showed that an (English) auction in which bidders offer fractions of the merged firm generates a higher expected profit for the target than any auction in which only cash is used. Crèmer (1987) has then pointed out that, with a combination of cash and shares, the target can extract virtually all gains from trade. Samuelson (1987) points out that these results hinge critically on there being no uncertainty about stand-alone values. Rhodes-Kropf and Viswanathan (2000) have considered different securities, and have shown that securities whose valuation is more sensitive to changes in the bidder's private information generate a higher expected revenue. Finally, 
DeMarzo, Kremer and Skrzypacz (2005) have also analyzed mechanisms in which only securities based on the value of the merged firm can be used. All these papers have focused on revenue maximizing mechanisms, occasionally allowing for moral hazard in the determination of the value of the merged firm. All these results however have been established under the assumption that the stand-alone values of all firms are known. ${ }^{6}$ Our results indicate that these findings are not robust to the introduction of private information about the firms' stand-alone values.

Our paper also contributes to the literature on mergers and acquisitions more broadly. ${ }^{7}$ Our work contrasts with recent work by Shleifer and Vishny (2003), in which managers of target firms knowingly accept overvalued shares because they think they can sell the shares before the market learns their true value. Their model hinges on market inefficiencies, whereas our analysis implicitly assumes that managers are reluctant to accept potentially over-valued shares. With uncertainty about stand-alone values and synergies, our model is reminiscent of the pioneering analysis of Myers and Majluf (1984). In fact, the classic Myers and Majluf analysis is a special case of our more general framework in which there is only one-sided asymmetric information. When asymmetric information is two-sided, as in our model, no pecking order emerges. In general, unlimited amounts of cash will not solve the two-sided asymmetric information problems we study.

The rest of the paper is organized as follows. Section 2 describes the model. In Section 3 we study the verifiable case, and Section 4 is devoted to the analysis of the non-verifiable case. Section 5 concludes. The appendix contains all proofs.

\footnotetext{
${ }^{6}$ Rhodes-Kropf and Viswanathan (2005) consider a second price auction in a model of mergers where bidders have information about both the joint value of the asset and the stand alone value of their firm.

${ }^{7}$ The theoretical literature on mergers and acquisitions is vast: see McAfee (1994), Rhodes-Kropf and Robinson (2005), Gort (1969), Jovanovic and Rousseau (2002), Maksimovic and Phillips (2001) or Gorton, Kahl and Rosen (2000) for a variety of treatments along different lines.
} 


\section{The Model}

In this section, we describe our formal model, which draws on the existing empirical evidence regarding the nature of interactions between firms in the merger market for its main features. In a sample of 400 large merger transactions in the 1990s, involving a total of over one trillion dollars in transaction value, Boone and Mulherin (2005) find that half are bilateral negotiations, while the remainder are structured as auctions. In the latter setting, a potential target is courted by an average of seven firms willing to sign confidentiality agreements. Of these seven firms, an average of about two submit private bids. Only after the winner of the private auction is announced does the merger event become public information to stock market participants.

To capture these features, we consider a setting in which a lone target firm can merge with exactly one of $n$ potential bidders. Because the existing empirical evidence suggests that around seven firms sign confidentiality agreements, which allow them to decide whether to enter a formal bid, we think it is important to consider a setting which is general enough to account for $n>1$. However, whenever the important features of our results can be highlighted within the single bidder framework, we will set $n=1$ to simplify exposition. For the most part Section 3 deals with the case of $n>1$, while Section 4 restricts attention to the single bidder case.

Formally, a target firm, which we label firm 0 , can merge with at most one of $n \geq 1$ potential partners, or 'bidders'. We denote the set of bidders by $N=\{1, \ldots, n\}$. It will also be convenient to define $N_{0}$ as the set of all $n+1$ firms, including the target. Our choice of the words 'bidder' and 'target' is simply for ease of exposition. (In Section 4 we use adverse selection arguments to identify who buys whom.) We assume that managers of the firm act in the interest of existing shareholders when they make their decisions, so that there is no distinction between a firm and its owner(s).

If firms 0 and $i$ merge, the total net worth of new firm is

$$
r_{i}=x_{0}+x_{i}+\gamma_{i}\left(w_{i}, v_{i}\right),
$$


where $x_{j}$ denotes firm $j$ 's the stand-alone value, and $w_{i}$ and $v_{i}$ denote the realizations of private signals observed by agent 0 and $i$ respectively. The function $\gamma_{i}$ specifies the synergy value, i.e. the amount by which the total value $r_{i}$ exceeds the sum of the two stand-alone values. This term can also be interpreted as the expected synergy, conditional on the signals $\left(w_{i}, v_{i}\right)$. We assume that $\gamma_{i}$ is additively separable and write, without additional loss of generality,

$$
\gamma_{i}\left(w_{i}, v_{i}\right)=w_{i}+v_{i}
$$

We also assume that all stand-alone values are privately known. Thus agent 0's privately known 'type' is the vector $\theta_{0}:=\left(x_{0}, w_{1}, \ldots, w_{n}\right)$; and for each $i \in N$, agent $i$ 's type is the pair $\theta_{i}:=\left(x_{i}, v_{i}\right)$.

Stand-alone values and synergy signals are all distributed independently, with respective supports $\left[\underline{x}_{j}, \bar{x}_{j}\right],\left[\underline{w}_{i}, \bar{w}_{i}\right]$, and $\left[\underline{v}_{i}, \bar{v}_{i}\right]$. The important part of this assumption is that the agents' types $\theta_{0}, \theta_{1}, \ldots, \theta_{n}$ are distributed independently. As is well known, under risk neutrality, even an arbitrarily small amount of correlation would remove the frictions created by private information (Crèmer and McLean (1988) and McAfee and Reny (1992)). ${ }^{8}$ The assumption that, for any firm, its stand-alone value and its signal(s) on synergies are independent is not essential and is made only to simplify the exposition. We denote the c.d.f. of $\theta_{i}$ by $F_{i}$ for each firm $i$.

We assume that $\underline{x}_{i}>0$ for each $i$, but do not rule out negative values for $w_{i}$ and $v_{i}$. However, to simplify the characterization of efficient merger outcomes, we assume that $\underline{x}_{0}+\underline{w}_{i}>0$ and $\underline{x}_{i}+\underline{v}_{i}>0$ for each $i$, so that the total contribution of a firm to a merger is non-negative.

We should point out that our model, while fairly general, leaves out some potentially interesting scenarios. For example, suppose that the $n+1$ firms compete in the same oligopolistic industry.

\footnotetext{
${ }^{8}$ Mechanisms that achieve full surplus extraction tend to be cumbersome and sensitive to the details of the information structure. Moreover, they require large payments when the correlation among types is small; thus they cannot be used in the presence of limited liability constraints or risk aversion. A full discussion of what kind of allocations can be achieved when types are correlated but risk aversion and/or limited liability are imposed is beyond the scope of this paper.
} 
In this case, when firms 0 and $i$ merge, the stand-alone value of the other bidders may also change because the increased concentration may lead to increased profits for all firms. Or conversely, the non-merged firms may be placed at a competitive disadvantage because the merged firm has superior pricing power. Furthermore, these changes may depend on the identity of the merging firms. In this paper we rule out this sort of identity-dependent externalities. (Note however that this is an an issue only when there is more than one potential bidder).

A feasible outcome in this environment consists of: i) a merger outcome, ii) a set of share redistribution, and iii) a set of monetary transfers, defined as follows:

1. A (stochastic) merger outcome is simply a probability distribution $p=\left(p_{0}, p_{1}, \ldots, p_{n}\right)$, where $p_{i}$ denotes the probability that firms 0 and $i$ merge. Because $p$ is a probability vector, its elements sum to one ( $p_{0}$ denotes the probability that no merger occurs).

2. A set of share redistributions consists of $n+1$ matrices $s=\left(s^{0}, s^{1}, \ldots, s^{n}\right)$. We consider arbitrary redistributions of shares, therefore each $s^{i}=\left[s_{j k}^{i}\right]$ is a square matrix of dimension $(n+1)$, in which entry $s_{j k}^{i} \in[0,1]$ denotes the fraction of firm $j$ 's value that goes to firm $k$ when firms 0 and $i$ merge. (In accord with the notation above, $s^{0}$ denotes the matrix of redistributions that take place when no merger occurs.) We adopt the convention that, when firm 0 and $i$ merge, the new firm takes the name of 'firm 0', and firm $i$ disappears. Thus the variables $s_{i j}^{i}, j \in N_{0}$ are irrelevant when $i \neq 0$. The total value in stocks given to agent $j$ is $s_{0 j}^{i} r_{i}+\sum_{k \neq i} s_{k j}^{i} x_{k}$ if firm 0 and $i$ merge, and $\sum_{k \in N_{0}} s_{k j}^{0} x_{k}$ if no merger occurs.

3. A set of monetary transfers is a matrix $t=\left(t^{0}, t^{1}, \ldots, t^{n}\right)$, where $t^{i}=\left(t_{0}^{i}, t_{1}^{i}, \ldots, t_{n}^{i}\right)$ and $t_{j}^{i}$ denotes the transfer to agent $j$ when firms 0 and $i$ merge $\left(t_{j}^{0}\right.$ denotes the cash transfer to firm $j$ when no merger occurs).

The object of our analysis is the set of all 'direct revelation mechanisms.' In the present context 
any such mechanism consists of three functions specifying: i) a merger outcome $p(\theta)$, ii) a set of share redistributions $s(\theta)$, and iii) a set of transfers $t(\theta)$, for any realization of the type profile $\theta:=\left(\theta_{0}, \theta_{1}, \ldots, \theta_{n}\right)$. With slight abuse of notation and terminology, we denote any mechanism by $\mu=(p, s, t)$, and call $p, s$ and $t$, the merger rule, the sharing rule and the transfer rule, respectively.

By the Revelation Principle, the outcome function of any equilibrium of any conceivable bargaining game among the $n+1$ firms corresponds to the truth-telling equilibrium outcome of a direct revelation mechanism. Thus by considering the set of all incentive-compatible revelation mechanisms we are not imposing any prior restriction on the set of negotiation protocols that the $n+1$ parties may want to engage in. We will focus on the efficient frontier of this set. Specifically, we will provide necessary and sufficient conditions for the implementability of efficient merger rules.

Since we do not want to restrict a priori the set of feasible mechanisms, we allow for the possibility of redistributing shares of non-merging firms. Although outcomes with this feature may appear unrealistic, our approach is motivated by the fact that some real-world contracts do allow for transfers among non-merging firms. For example, preliminary merger agreements sometimes include 'break-up fees', stating that the target has to make payments to a losing bidder. ${ }^{9}$ Therefore, it seems reasonable a priori to think that transfers between non-merging firms may occur, and arbitrarily restricting contracts to exclude these kind of payments seems unwarranted.

Indeed, we will show that avoiding the redistribution of shares of non-merging firms is optimal; i.e., it is never necessary to redistribute shares of non-merging firms to implement the efficient merger rule. In fact, in all cases in which the efficient merger rule is non-trivial, redistributing the shares of non-merging firms undermines efficiency. Intuitively, this is because non-merging firms remain privately informed about their stand-alone values; and thus transferring their shares to other firms necessarily entails the payment of information rents. Instead of simply restricting

\footnotetext{
${ }^{9}$ Che and Lewis (2003) discuss economic rationales for break-up fees and other contractual arrangements in mergers.
} 
the set of feasible merger contracts a priori, we arrive at the exclusion of these transfers by using optimization arguments.

Our main goal is to provide necessary and sufficient conditions for the implementability of incentive-efficient merger rules. In other words, given an efficient rule $p$, we look for the existence of a feasible sharing rule $s$ and a budget-balanced transfer rule $t$ such that the mechanism $\mu=(p, s, t)$ is incentive-feasible: in the revelation game induced by $\mu$, truth-telling forms a Bayesian-Nash equilibrium (i.e. $\mu$ is incentive compatible) and yields nonnegative expected surplus for all types of all agents ( $\mu$ is individually rational).

The budget balance requirement entails the following constraints:

$$
\sum_{k=0}^{n} s_{j k}^{i}(\theta) \leq 1 \text { and } \sum_{j=0}^{n} t_{j}^{i}(\theta) \leq 0 \quad \text { for all } i, j \in N_{0}, \theta \in \Theta
$$

Moreover, since we focus on efficient mechanisms, in equilibrium condition (3) will hold with equality. The possibility of 'wasting' resources, i.e. transferring them to a third party, is useful off the equilibrium path, in order to punish reports which are incompatible with observed merger values, but will not occur on the path of an equilibrium we consider. ${ }^{10}$

From now on we will say that a mechanism $\mu$ is budget-feasible when condition (3) holds, with equality in equilibrium; and that $\mu$ is incentive-feasible when it is budget-feasible and satisfies incentive compatibility and individual rationality.

The net surplus that agent $j$ expects from participating in the mechanism $\mu=(p, s, t)$ can be written as

$$
u_{j}\left(\theta^{\prime}, \theta\right) \equiv p_{0}\left(\theta^{\prime}\right)\left[\sum_{i \in N_{0}} s_{i j}^{0}\left(\theta^{\prime}\right) x_{i}+t_{j}^{0}\left(\theta^{\prime}\right)\right]
$$

$$
+\sum_{i \in N} p_{i}\left(\theta^{\prime}\right)\left[s_{0 j}^{i}\left(\theta^{\prime}\right) r_{i}+\sum_{k \neq i} s_{k j}^{i}\left(\theta^{\prime}\right) x_{k}+t_{j}^{i}\left(\theta^{\prime}\right)\right]-x_{j}
$$

\footnotetext{
${ }^{10}$ This point will be clarified below when we discuss mechanism design with verifiable merger values.
} 
where $\theta^{\prime}$ and $\theta$ denote the profile of reported and true types, respectively, and the values $r_{1}, \ldots, r_{n}$ and $x_{0}, . ., x_{n}$ are determined by the vector $\theta$. (Recall that $r_{i}=x_{0}+x_{i}+w_{i}+v_{i}$.) The first term is the value of transfers - both through direct cash payments as well as any arbitrary reshuffling of ownership - that the agent receives when no merger occurs. The second term captures the expected surplus accruing to bidder $j$ when mergers occur, given that a merger may or may not involve bidder $j$. The last term, $-x_{j}$, is there to normalize payoffs so that non-participation yields zero net surplus.

The social surplus generated by $\mu$ can be written as

$$
\sum_{j \in N_{0}} u_{j}\left(\theta^{\prime}, \theta\right)=\sum_{i \in N} p_{i}\left(\theta^{\prime}\right)\left(r_{i}-x_{0}-x_{i}\right)=\sum_{i \in N} p_{i}\left(\theta^{\prime}\right)\left(w_{i}+v_{i}\right)
$$

The interim expected surplus of type $\theta_{j} \in \Theta_{j}$ of agent $j$, when all other agents report their true types, and agent $j$ reports $\theta_{j}^{\prime}$, is

$$
U_{j}\left(\theta_{j}^{\prime}, \theta_{j}\right) \equiv \int_{\Theta_{-j}} u_{j}\left(\left(\theta_{j}^{\prime}, \theta_{-j}\right),\left(\theta_{j}, \theta_{-j}\right)\right) d F_{-j}\left(\theta_{-j}\right)
$$

Since $u_{j}\left(\theta^{\prime}, \theta\right)$ was defined as net surplus (i.e. after subtracting the stand alone value $x_{j}$ ), the interim individual rationality constraint can be written as $U_{j}\left(\theta_{j}, \theta_{j}\right) \geq 0$ for each $j$, and $\theta_{j}$.

Whether a certain mechanism satisfies incentive compatibility depends on the information available to the agents and to the mechanism designer. In particular, the set of incentive-feasible mechanisms differs depending on whether new information becomes available after a merger.

The recent literature on mechanism design with interdependent values has emphasized the importance of using information generated after the decision is taken to determine monetary transfers. Mezzetti (2002) has pointed out that, with interdependent values, asking each agent to report the utility generated by the decision provides valuable information, since such utility depends on the types of the other agents. The reported utility values can be compared with the values that should have been observed given the initial reports by the agents, and thus the transfers can be made contingent on both the ex ante reported types and the ex post reported utilities. For the quasi-linear 
case, Mezzetti has shown that, using a two-stage mechanism, it is always possible to construct budget-balanced and incentive compatible mechanisms that implement efficient decision rules (although individual rationality may not be satisfied; additional conditions are necessary). On the other hand, Jehiel and Moldovanu (2001) have previously shown that, when only ex ante information can be used, with interdependent values it is generally impossible to implement efficient decision rules. ${ }^{11}$

We will analyze two cases:

1. Verifiable merger values. The designer can make both share redistributions and monetary payments contingent on the realized total value of any new firm, as well as on the agents' reports about their types. We assume that no information about the stand-alone value of each non-merging firm becomes available after a merger.

2. No after-merger information. In this case share redistributions and monetary payments can only depend on the agents' reports, and thus the mechanism design problem is the classic, static one.

Of course, the empirical reality behind mergers and acquisitions lies somewhere between these two extremes. Firms often use contractual features such as collars, earn-outs or contingent value rights as part of merger considerations. These contractual features help to tie the value of the consideration to the ex post value of the newly formed firm. At the same time, ex post litigation is a widely used tool to levy punishments when mergers are later thought to have been predicated on false information. The ability to appeal to the legal system for remedies when false reports occur highlights the tension between these two polar extremes: on the one hand, the legal system provides

\footnotetext{
${ }^{11}$ Neither Mezzetti nor Jehiel and Moldovanu consider the possibility of transferring value through ownership redistributions.
} 
punishments and remedies when information used to make a merger decision is later shown to be false; on the other hand, the system is costly and not without error.

The assumption of verifiable merger values in the first case is made only for simplicity. The crucial assumption is the possibility of writing contracts that are contingent on information that becomes available after the merger. If the value of any new firm were not verifiable but merely observable by each merging partner, we could use a two-stage mechanism a la Mezzetti to make transfers depend on the realized value of the merger. The assumption of verifiability simplifies the exposition since it allows the agents to write contracts in which transfers are contingent on the realized value of the merger, without any need for a second round of messages.

It is also worth noticing that we treat differently merging and non-merging firms, and in particular we assume that the stand-alone value of non-merging firms is never observable. There are several reasons why it is reasonable to assume that the stand-alone value of non-merged firms is unverifiable. Recent models of mergers and acquisitions such as Rhodes-Kropf and Viswanathan (2005) and Shleifer and Vishny (2003) assume that a firm's 'true' stand-alone value may differ than the price observed in markets because managers may possess inside information about future business plans that it is not known to market participants. Even if managers were to make forwardlooking statements about business plans under 'safe harbor' provisions, these statements would be largely unverifiable since it would be difficult to distinguish between unexpected failure to meet expectations from untruthful reports.

\section{$3 \quad$ Verifiable Merger Values}

This section is devoted to the analysis of the case in which transfers can be conditioned on ex post observables. In particular, we assume that the value of any newly formed firm can be verified ex post, while it remains impossible to verify the stand-alone value of firms not involved in the merger. 
Our analysis here ultimately delivers bad news for merger efficiency. We establish necessary and sufficient conditions for the implementation of an efficient merger rule (Proposition 1), and show that, if there is only one bidder, the condition cannot be satisfied whenever it is not common knowledge that the merger is always value-enhancing (Corollary 1). We also identify a condition on the support of all firms types under which, no matter how many firms are involved in the negotiations, the first-best cannot be achieved (Corollary 2), and show that when this condition is violated the first-best can be achieved for a sufficiently large number of bidders.

It is worth emphasizing here that private information about both stand alone and synergy values can generate significant distortions from the first best, even though the ex post verifiability of any newly created firm makes its shares and cash equivalent means of payment. Thus, even when the cards are stacked in favor of efficiency, the presence of hidden information causes some of the gains from merger to remain unrealized in any conceivable equilibrium in the M\&A market. Not only does the presence of private information cause 'too few' mergers to occur in equilibrium, in the sense that mergers with small but positive synergies go unrealized, but the 'wrong' mergers often occur, in the sense that the optimal acquirer in the presence of informational constraints is not always the acquirer that produces the largest synergy values.

We arrive at the main result (Proposition 1) in a series of steps. Lemma 1 establishes the equivalence of stock and cash when the value of the newly merged firm is verifiable ex post. Based on this equivalence, we derive a critical result (Lemma 4): any implementable merger rule can also be implemented without redistributing the shares of the firms not involved in the merger. In fact, in all interesting cases (i.e., whenever the merger rule is non-trivial), it is suboptimal to redistribute shares of non-merging firms. This 'leaving losers untouched' result enables us to show that, under a suitably chosen rule for allocating the shares of the new firm (defined in condition (23) below) the general design problem can be mapped into a private value framework. This in turn allows us to apply well known conditions, derived by Williams (1999) and Krishna (2002), for 
the implementability of efficient social decision rules.

\subsection{The Equivalence of Stock and Cash}

Given the multiplicity of ways in which payments can be made (using combinations of stock and cash transfers), it is natural to begin the analysis by asking whether it is possible to restrict the class of mechanisms that we need to consider. Our first lemma establishes that neither the set of implementable merger rules, nor the distribution of the total surplus among the agents that is generated by any merger rule depend on how the shares of any new firm are allocated. This is because the total value of any new firm is verifiable, hence its shares are equivalent to money. Recall that $s_{j k}^{i}$ denotes the fraction of firm $j$ 's shares that are transferred to firm $k$ in the event that firms 0 and $i$ merge, and let $s_{j .}^{i}:=\left(s_{j 0}^{i}, s_{j 1}^{i}, \ldots, s_{j n}^{i}\right)$.

Lemma 1 For any budget-feasible mechanism $(p, s, t)$, and any sharing rule $\widetilde{s}$ such that

$$
\widetilde{s}^{0}=s^{0} \text { and } \widetilde{s}_{j .}^{i}=s_{j}^{i} \text { for all } i \in N, j \in N_{0} \backslash\{i\},
$$

there exists a feasible transfer rule $\widetilde{t}$ such that the new mechanism $(p, \widetilde{s}, \widetilde{t})$ generates the same ex post expected surplus functions $u_{j}$, defined in (4), for all firms $j \in N_{0}$.

The equivalence property established in Lemma 1 can be restated as follows. Consider any mechanism $(p, s, t)$, and any sharing rule $\widetilde{s}$ that allocates the shares of all non-merging firms as $s$ does, and the shares of any new firm arbitrarily (that is, we may have $\widetilde{s}_{0}^{i} \neq s_{0}^{i}$.). Then there exists a transfer rule $\widetilde{t}$ such that the mechanism $(p, \widetilde{s}, \widetilde{t})$ generates an equivalent revelation game, in the sense that the payoff functions of all agents are the same. Therefore, if the original mechanism $(p, s, t)$ satisfies incentive compatibility and individual rationality, so does the new mechanism $(p, \widetilde{s}, \widetilde{t})$. Note that the equivalence between stock and cash has been established only for the shares of any new firm. As we will see next, the equivalence does not extend to the shares of non-merging firms, essentially because their value is not verifiable ex post. 


\subsection{Leaving Losers Untouched}

A natural question in light of Lemma 1 is whether the neutrality of the ownership structure of any new firm holds for non-merging firms as well. In particular, is there any loss of generality in restricting attention to mechanisms that leave all shares of each non-merging firm with its original owner? Formally, we say that a sharing rule $s$ satisfies the 'Losers Untouched' (LU) property if

$$
s_{k k}^{0}(\theta)=s_{j j}^{i}(\theta)=1 \text { for all } k \in N_{0}, i, j \in N \text {, all } \theta .
$$

If it were the case that any implementable merger rule $p$ could also be implemented with a mechanism $(p, s, t)$ where $s$ satisfies the LU property, then the extra flexibility given by the possibility of redistributing shares through $s$ would be of no value, and we could narrow down the design problem, without loss of generality, to the specification of monetary transfers.

It turns out that the way shares of non-merging firms are allocated does affect the distribution of the total surplus generated by any merger rule among the agents, and thus affects the set of merger rules that can be implemented. This is because the stand-alone value of each non-merging firm remains unverifiable ex post; therefore incentive rents have to be paid in order to elicit truth-telling. We will show, as an immediate implication of our next lemma, that the expected information rent earned by agent $i$ depends negatively on the fraction $s_{i i}^{j}$ of firm $i$ that he or she retains when his or her firm does not merge. Therefore, changing $s_{i i}^{j}$ changes the distribution of the total surplus generated by any given merger rule.

However, we will also show that a merger rule is $L U$-implementable - i.e. can be implemented with an incentive-feasible mechanism $(p, s, t)$ where $s$ satisfies the LU property - if and only if it satisfies a set of monotonicity conditions, spelled out in (20) below; and it will be straightforward to check that these monotonicity conditions are satisfied by any efficient merger rule (defined formally in (22) below).

The derivation of these results hinges on the fact that, due to ex post verifiability, the type of 
each agent becomes one-dimensional in equilibrium. Feasible mechanisms can involve large fines whenever the agents report types that are inconsistent with the total value of any new firm. Since each bidder $i$ expects the target to report its true type, it cannot be optimal for him to lie about the total $x_{i}+v_{i}$ of its two pieces of private information, unless the probability of his firm merging conditional on his report is zero, in which case the lie would go undetected.

Similarly, since the target expects truthful reporting by all potential bidders, it cannot be optimal for her to lie about any total $x_{0}+w_{i}$, unless there is no chance of merging with firm $i$ conditional on this report. Thus, unless the report $\theta_{i}^{\prime}$ implies that firm $i$ has no chance of merging, agent $i$ must issue a report in which the sum total of stand-alone values and synergies are correctly reported: each agent can misreport the fraction of total surplus due to synergies, but not the overall value. $^{12}$

We now move to the formal analysis. The set of undetectable lies ${ }^{13}$ for any type $\theta_{i}$ of bidder $i$ is given by

$$
\mathcal{L}_{i}\left(\theta_{i}\right):=\left\{x_{i}^{\prime}+v_{i}^{\prime}=\tau_{i}\right\}
$$

where $\theta_{i}=\left(x_{i}, v_{i}\right)$ and $\tau_{i}=x_{i}+v_{i}$. Similarly, since a target of type $\theta_{0}=\left(x_{0}, w_{1}, \ldots, w_{n}\right)$ may report wrong values $\left(x_{0}, w_{i}\right)$ only if $x_{0}^{\prime}+w_{i}^{\prime}=x_{0}+w_{i}$, we can define the set of undetectable lies by the target firm for any $\theta_{0} \in \Theta_{0}$ as

$$
\mathcal{L}_{0}\left(\theta_{0}\right):=\left\{\theta_{0}^{\prime} \in \Theta_{0} \mid x_{0}^{\prime}+w_{i}^{\prime}=\sigma_{i} \text { each } i \in N\right\}
$$

\footnotetext{
${ }^{12}$ It is also worth noting that the incentive problems arise precisely because of the ex post unverifiability of standalone values $x_{i}$. If $x_{i}$ were to become observable in a second stage, then large fines could be prescribed for a firm reporting $\widehat{x}_{i} \neq x_{i}$. This would prevent agent $i$ from announcing any $\widehat{x}_{i} \neq x_{i}$ for which the probability of merging is not one; and all incentive problems would essentially disappear.

${ }^{13}$ In all mechanisms that are relevant to our purposes, no agent can have any incentive to lie issuing a report that ensures a zero probability of merger, as any such report yields zero expected surplus. Therefore we ignore such reports in what follows.
} 
where $\sigma_{i}:=x_{0}+w_{i}$. Note that each set in (8) and (9) is one-dimensional. Each realization of $\underline{x}_{0}+\underline{w}_{i}$ and $\underline{x}_{i}+\underline{v}_{i}$ corresponds to a separate mechanism design problem. The relevant incentive compatibility constraints for each bidder $i \in N$ and for the target can now be written as

$$
U_{i}\left(\theta_{i}, \theta_{i}\right) \geq U_{i}\left(\theta_{i}^{\prime}, \theta_{i}\right), \quad \theta_{i}^{\prime} \in \mathcal{L}_{i}\left(\theta_{i}\right)
$$

and

$$
U_{0}\left(\theta_{0}, \theta_{0}\right) \geq U_{0}\left(\theta_{0}^{\prime}, \theta_{0}\right), \quad \forall \theta_{0}^{\prime} \in \mathcal{L}_{0}\left(\theta_{0}\right)
$$

To see that redistributing shares of non-merging firms generates information rents for their original owners, it is useful to redefine the target's type as

$$
\theta_{0}:=\left(x_{0}, \sigma_{1}, \ldots, \sigma_{n}\right)
$$

where $\sigma_{i}:=x_{0}+w_{i}$, and each of the bidder's types as

$$
\theta_{i}:=\left(x_{i}, \tau_{i}\right),
$$

where $\tau_{i}:=x_{i}+v_{i}$. For each bidder $i$ and each $\tau_{i} \in\left[\underline{x}_{i}+\underline{v}_{i}, \bar{x}_{i}+\bar{v}_{i}\right]$, let $\left[\underline{x}^{\tau_{i}}, \bar{x}^{\tau_{i}}\right]$ denote the interval of values of $x_{i}$ that correspond to the line $\mathcal{L}_{i}\left(x_{i}, \tau_{i}\right)$, defined in (8); that is,

$$
\left[\underline{x}^{\tau_{i}}, \bar{x}^{\tau_{i}}\right]:=\left\{x_{i} \in\left[\underline{x}_{i}, \bar{x}_{i}\right] \mid \exists v_{i} \in\left[\underline{v}_{i}, \bar{v}_{i}\right] \text { such that } x_{i}+v_{i}=\tau_{i}\right\}
$$

and define

$$
B_{i}^{\tau_{i}}(\zeta) \equiv 1-E_{\theta_{-i}}\left[\sum_{j \in N_{0} \backslash\{i\}} p_{j}\left(\left(\zeta, \tau_{i}\right), \theta_{-i}\right) s_{i i}^{j}\left(\left(\zeta, \tau_{i}\right), \theta_{-i}\right)\right], \quad \zeta \in\left[\underline{x}^{\tau_{i}}, \bar{x}^{\tau_{i}}\right]
$$

and

$$
U_{i}^{\tau_{i}}(\zeta) \equiv U_{i}\left(\left(\zeta, \tau_{i}\right),\left(\zeta, \tau_{i}\right)\right), \quad \zeta \in\left[\underline{x}^{\tau_{i}}, \bar{x}^{\tau_{i}}\right]
$$

In words, $B_{i}^{\tau_{i}}(\zeta)$ is the fraction of its own stand-alone value that firm $i$ expects to give away when losing the merger contest, and $U_{i}^{\tau_{i}}(\zeta)$ is the interim expected utility, for type $\tau_{i}$ of firm $i$, when reporting $\zeta$ from the set of undetectable lies given by equation (8). 
Similarly, for each $\sigma=\left(\sigma_{1}, \ldots, \sigma_{n}\right)$, let $\left[\underline{x}_{0}^{\sigma}, \bar{x}_{0}^{\sigma}\right]$ denote the interval of values of $x_{0}$ that correspond to the segment $\mathcal{L}_{0}\left(x_{0}, \sigma\right)$, defined in (9), i.e.

$$
\left[\underline{x}_{0}^{\sigma}, \bar{x}_{0}^{\sigma}\right]:=\left\{x_{0} \in\left[\underline{x}_{0}, \bar{x}_{0}\right] \mid \exists\left(w_{1}, \ldots, w_{n}\right) \in \times_{i \in N}\left[\underline{w}_{i}, \bar{w}_{i}\right] \text { such that } x_{0}+w_{i}=\sigma_{i}, i \in N\right\}
$$

and define

$$
B_{0}^{\sigma}(\zeta) \equiv 1-E_{\theta_{-0}}\left[p_{0}\left((\zeta, \sigma), \theta_{-0}\right) s_{00}^{0}\left((\zeta, \sigma), \theta_{-0}\right)\right], \quad \zeta \in\left[\underline{x}_{0}^{\sigma}, \bar{x}_{0}^{\sigma}\right]
$$

and

$$
U_{0}^{\sigma}(\zeta) \equiv U_{0}((\zeta, \sigma),(\zeta, \sigma)), \quad \zeta \in\left[\underline{x}_{0}^{\sigma}, \bar{x}_{0}^{\sigma}\right]
$$

As before, $B_{0}^{\sigma}(\zeta)$ is the fraction of her stand-alone value that the type $\sigma$ of firm 0 expects to give up when no merger takes place as a result of issuing a report $\zeta$, and $U_{0}^{\sigma}(\zeta)$ is the utility associated with that report. The next lemma is based on standard mechanism design arguments. The proof is provided in the appendix, for the sake of completeness.

Lemma 2 The incentive compatibility constraints in (11) imply that $B_{0}^{\sigma}$ is non-increasing and

$$
U_{0}^{\sigma}\left(x_{0}\right)=U_{0}^{\sigma}\left(\bar{x}_{0}^{\sigma}\right)+\int_{x_{0}}^{\bar{x}_{0}^{\sigma}} B_{0}^{\sigma}(\zeta) d \zeta, \quad \text { for all } x_{0} \in\left[\underline{x}_{0}^{\sigma}, \bar{x}_{0}^{\sigma}\right]
$$

The incentive compatibility constraints in (10) imply that, for each $i \in N, B_{i}^{\tau_{i}}$ is non-increasing and

$$
U_{i}^{\tau_{i}}\left(x_{i}\right)=U_{i}^{\tau_{i}}\left(\bar{x}_{i}^{\tau_{i}}\right)+\int_{x_{i}}^{\bar{x}_{i}^{\tau_{i}}} B_{i}^{\tau_{i}}(\zeta) d \zeta, \text { for all } x_{i} \in\left[\underline{x}^{\tau_{i}}, \bar{x}^{\tau_{i}}\right]
$$

Lemma 2 has two important implications. First, the 'envelope condition' in (18) shows that the information rent of each type $\left(x_{i}, \tau_{i}\right)$ of agent $i$ is determined by the function $B_{i}^{\tau_{i}}$. Since $B_{i}^{\tau_{i}}$ decreases with the fraction $s_{i i}^{j}$ of firm $i$ that agent $i$ retains whenever his firm does not merge, his information rent depends negatively on $s_{i i}^{j}$. Similarly, the envelope condition in (17) shows that the target's information rent decreases with the fraction of her firm retained when no merger takes 
place. Therefore, in order to minimize information rents, the shares of all non-merging firm should never be redistributed.

Second, if the LU property holds, the functions defined in (16) and (15) become

$$
B_{0}^{\sigma}(\cdot)=1-P_{0}(\cdot, \sigma), \quad \text { and } B_{i}^{\tau_{i}}(\cdot)=P_{i}\left(\cdot, \tau_{i}\right)
$$

where $P_{0}\left(x_{0}, \sigma\right) \equiv E_{\theta_{-0}}\left[p_{0}\left(\left(x_{0}, \sigma\right), \theta_{-0}\right)\right]$ is the probability that no merger occurs conditional on firm 0 announcing type $\left(x_{0}, \sigma\right)$ and $P_{i}\left(x_{i}, \tau_{i}\right) \equiv E_{\theta_{-i}}\left[p_{i}\left(\left(x_{i}, \tau_{i}\right), \theta_{-i}\right)\right]$ is the probability that firm $i$ merges, conditional on its owner announcing $\left(x_{i}, \tau_{i}\right)$. Since by Lemma 2 incentive compatibility implies that both $B_{0}^{\sigma}$ and $B_{i}^{\tau_{i}}$ are non-increasing, the following monotonicity conditions are necessary for any (implementable) merger rule $p$ to be LU-implementable:

$$
\text { for each } \sigma \in \times_{i \in N}\left[\underline{x}_{0}+\underline{w}_{i}, \bar{x}_{0}+\bar{w}_{i}\right], \quad P_{0}(\cdot, \sigma) \text { is non-decreasing }
$$

$$
\text { for each } i \in N, \tau_{i} \in\left[\underline{x}_{i}+\underline{v}_{i}, \bar{x}_{i}+\bar{v}_{i}\right], \quad P_{i}\left(\cdot, \tau_{i}\right) \text { is non-increasing. }
$$

The next result establishes that these monotonicity conditions are also sufficient for LU-implementability with any mechanism that does not require outside financing, i.e.

$$
\sum_{i=0}^{n} t_{i}^{j}(\theta) \leq 0, \text { for all } \theta \in \Theta \text { and all } j \in N_{0} \text {. }
$$

Intuitively, this is because, as (17) and (18) show, applying the LU property can only reduce the information rent of any type of any agent, and thus can only make it easier to satisfy both budget balance and individual rationality.

Lemma 3 Suppose that $(p, s, t)$ is incentive-feasible, $p$ satisfies the monotonicity conditions in (20) and $t$ satisfies (21). Then there exists an incentive-feasible mechanism $(p, \widetilde{s}, \widetilde{t})$ where $\widetilde{s}$ satisfies the $L U$ property and $\widetilde{t}$ satisfies (21). 
The proof of Lemma 3 is by construction: the new transfer rule $\widetilde{t}$ is designed so that the change in the total value received (both in shares and cash) by each type of each agent, for each realization of the opponents' types, generates the lowest information rent that is consistent with the incentive compatibility and individual rationality constraints.

\subsection{Implementing Efficient Merger Rules}

The preceding analysis establishes that the information rent accruing to an agent is independent of the sharing rule that is chosen for the merged firm. Consider now the efficient merger rule $p^{*}:=\left(p_{0}^{*}, \ldots, p_{n}^{*}\right)$, defined as

$$
p_{i}^{*}(\theta)= \begin{cases}1, & \text { if } v_{i}+w_{i}>\max \left\{0, v_{j}+w_{j} ; j \neq i\right\}, \\ 0, & \text { otherwise. }\end{cases}
$$

It is straightforward to check that $p^{*}$ satisfies the monotonicity conditions in (20). Thus, Lemma 3 immediately implies the following result.

Lemma 4 If the efficient rule $p^{*}$ is implementable by a mechanism that satisfies (21), it is also LU-implementable.

The goal of this subsection is to establish necessary and sufficient conditions under which the efficient rule $p^{*}$ is implementable. Establishing necessary conditions for implementability is relatively straightforward: by Lemma 4, we can restrict attention without loss of generality to mechanisms satisfying the LU property. Furthermore, Lemma 1 implies that we can allocate the shares of any new firm arbitrarily. Standard mechanism design arguments imply that the necessary conditions for the implementability of $p^{*}$ are satisfied. In particular, combining the incentive compatibility, individual rationality, and ex post budget balance constraints, we derive the inequality in Proposition 1 below, which essentially states that social surplus must exceed the sum of all information rents that have to be paid under the merger rule $p^{*}$. 
Sufficient conditions for the existence of a mechanism implementing $p^{*}$ are more difficult to establish. We are in a world of interdependent values, where the utility of any agent is affected by the types of the other agents. However, by judicious choice of the sharing rule, we can transform our interdependent values problem into a private value problem. This allows us to use the results of Williams (1999) and Krishna (2002) to establish sufficient conditions for $p^{*}$. And since the necessary conditions for implementability hold irrespective of the sharing rule, we obtain necessary and sufficient conditions for the existence of efficient merger rules.

Consider the following mechanism. Let $\sigma_{i}=x_{0}+w_{i}$ and $\tau_{i}=x_{i}+v_{i}$ be the true sums of stand-alone values and synergies under the proposed merger, and for reports $\widehat{\theta}_{0}=\left(\widehat{x}_{0}, \widehat{w}_{1}, \ldots, \widehat{w}_{n}\right)$ and $\widehat{\theta}_{i}=\left(\widehat{x}_{i}, \widehat{v}_{i}\right)$ let $\widehat{\sigma}_{i}=\widehat{x}_{0}+\widehat{w}_{i}$ and $\widehat{\tau}_{i}=\widehat{x}_{i}+\widehat{v}_{i}$. The mechanism uses the reported values and the ex post observed values to determine shares and payments as follows:

- If firm 0 and $i$ merge and the observed value $\sigma_{i}+\tau_{i}$ is different from the one announced by the firms, then all firms pay large penalties.

- If $\sigma_{i}+\tau_{i}=\widehat{\sigma}_{i}+\widehat{\tau}_{i}$ then the sharing rule $s$ is determined by the LU property, and by

$$
s_{00}^{i}(\theta)=1-s_{0 i}^{i}(\theta)=\frac{\widehat{\sigma}_{i}}{\widehat{\sigma}_{i}+\widehat{\tau}_{i}}
$$

Note that the denominator in equation (23) is positive because $\underline{x}_{0}+\underline{w}_{i}>0$ and $\underline{x}_{i}+\underline{v}_{i}>0$. In the truth-telling equilibrium of the mechanism, when the target and bidder $i$ merge, the target gets fraction $\frac{\sigma_{i}}{\sigma_{i}+\tau_{i}}$ of the new firm, and bidder $i$ gets the remaining fraction $\frac{\tau_{i}}{\sigma_{i}+\tau_{i}}$; the shares of all other firms are left untouched. Note that, by Lemma 1, there is no loss of generality in analyzing the implementation problem by fixing the shares in this way, since the choice of sharing rule plays no role in the necessary conditions for the existence of the efficient merger rule.

Under this sharing rule, the ex post expected payoff functions, when $\sigma_{i}$ and $\tau_{i}$ are reported truthfully (while the components of the sums $x_{0}+w_{i}$ and $x_{i}+v_{i}$ may be misreported) can be 
written as

$$
u_{0}\left(\theta^{\prime} ; \theta\right)=\sum_{i \in N} p_{i}\left(\theta^{\prime}\right) w_{i}+\sum_{j \in N_{0}} p_{j}\left(\theta^{\prime}\right) t_{0}^{j}\left(\theta^{\prime}\right)
$$

for firm 0 and as

$$
u_{i}\left(\theta^{\prime} ; \theta\right)=p_{i}\left(\theta^{\prime}\right) v_{i}+\sum_{j \in N_{0}} p_{j}\left(\theta^{\prime}\right) t_{i}^{j}\left(\theta^{\prime}\right)
$$

for each firm $i \in N$. The important point here is that the payoff function of each agent $j \in N_{0}$ is now independent of the other agents' types.

To complete the transformation of the model into a classic mechanism design environment with private values, we let the variable $a$ denote any deterministic merger outcome, so that the social surplus generated by decision $a$, when the type profile is $\theta$, can be written as

$$
S(a, \theta) \equiv \sum_{j \in N_{0}} \widetilde{v}_{j}\left(a, \theta_{i}\right)
$$

where

$$
\widetilde{v}_{0}\left(a, \theta_{0}\right)=\left\{\begin{array}{ccc}
0, & \text { if } \quad a=0, \\
w_{i}, & \text { if } \quad a=i ;
\end{array} \quad \widetilde{v}_{i}\left(a, \theta_{i}\right)=\left\{\begin{array}{c}
0, \quad \text { if } a \neq i, \\
v_{i}, \quad \text { if } a=i,
\end{array}\right.\right.
$$

and the ex post expected payoff functions of each agent can be rewritten as

$$
u_{j}(a, \theta)=\widetilde{v}_{j}\left(a, \theta_{j}\right)+t_{j}, \quad j \in N_{0} .
$$

Since $S(0, \theta)=0$ and $S(i, \theta)=v_{i}+w_{i}$, any rule $a^{*}$ that satisfies

$$
a^{*}(\theta) \in \arg \max _{a \in N_{0}} S(a, \theta)
$$

corresponds to the ex post efficient merger rule in the sense that $p_{j}^{*}(\theta)=1$ if and only if $j=a^{*}(\theta) .{ }^{14}$

\footnotetext{
${ }^{14}$ Ties are zero probability events, thus we can apply any arbitrary tie-breaking rule. For example, if $a^{*}(\theta)$ has multiple elements we select the element with the highest index.
} 
The problem of finding a transfer rule for which $p^{*}$ is implementable is now essentially a special case of more general models with private values studied in Williams (1999) and Krishna (2002). Williams provides a necessary and sufficient condition for the implementability of efficient decision rules with Groves mechanisms that satisfy ex ante budget balance. Here we simply observe that for any mechanism with transfers satisfying the weak budget balance condition (21) we can find a corresponding mechanism satisfying ex ante budget balance (it is sufficient to add appropriate positive constants to the transfers). Thus Lemma 3 applies, and we can restrict attention without loss of generality to mechanisms satisfying the LU rule. Finally, Krishna establishes the equivalence, in terms of interim expected surplus functions, of Groves mechanisms satisfying ex ante budget balance with interim incentive compatible mechanisms satisfying ex post budget balance.

To formalize this argument, first define

$$
\underline{Z}_{j}^{(\sigma, \tau)}:=\inf _{y \in \widetilde{\Theta}_{-j}(\sigma, \tau)} E_{\theta_{-j}}\left[\widetilde{v}_{j}\left(a^{*}\left(y, \theta_{-j}\right), y\right)+\sum_{k \in N_{0} \backslash j} \widetilde{v}_{k}\left(a^{*}\left(y, \theta_{-j}\right), \theta_{k}\right)\right], \quad j \in N_{0},
$$

where $\widetilde{\Theta}_{-0}(\sigma, \tau)$ denotes the support of the distribution of $\left(v_{1}, \ldots, v_{n}\right)$ conditional on $\sigma$ and $\tau$, and $\widetilde{\Theta}_{-i}(\sigma, \tau)$ denotes the support of the distribution of $\left(w_{1}, \ldots, w_{n}, v_{1}, \ldots, v_{i-1}, v_{i+1}, \ldots, v_{n}\right)$ conditional on $\sigma$ and $\tau$. We have the following result.

Proposition 1 The efficient merger rule $p^{*}$ is implementable with an ex post budget-balanced mechanism if and only if for all realizations of $\sigma, \tau$ we have

$$
n E_{\theta}\left[\sum_{i \in N_{0}} \widetilde{v}_{i}\left(a^{*}(\theta), \theta_{i}\right)\right] \leq \sum_{i \in N_{0}} \underline{Z}_{i}^{(\sigma, \tau)} .
$$

This condition completely characterizes the environments in which the implementation of efficient merger rules is possible, but it is not immediately intuitive. To unpack the intuition behind condition (29), first observe that the inequality in condition (29) is equivalent to the following four conditions: (i) efficiency, (ii) incentive compatibility, (iii) individual rationality, and (iv) budget 
balance. As highlighted in Williams (1999), the class of all efficient and interim incentive compatible mechanisms is equivalent in terms of surplus distribution to the set of Groves mechanisms. In other words, for any $\left(p^{*}, t\right)$, there is an equivalent Groves mechanism, which we can denote $\left(p^{*}, t^{G}\right)$, where $t^{G}$ equals

$$
t_{i}^{G}=\sum_{j \neq i} \tilde{v}_{j}\left(a^{*}, \theta_{j}\right)-h_{i}\left(\theta_{-i}\right)
$$

where the $h_{i}$ is an arbitrary 'entry-fee' for agent $i$. To achieve budget balance, the sum of all agents' entry fees must at least equal $n$ times the social surplus. This is because the standard logic of Groves mechanisms creates the merged firm only once but requires every agent to receive the social surplus. But the individual rationality constraint requires that each agent's entry fee does not exceed the interim expected surplus of his worst-off type. Combining these constraints yields

$$
n E_{\theta}\left[\sum_{i \in N_{0}} \widetilde{v}_{i}\left(a^{*}(\theta), \theta_{i}\right)\right] \leq \sum_{i \in N_{0}} h_{i}\left(\theta_{-i}\right) \leq \sum_{i \in N_{0}} \underline{Z}_{i}^{(\sigma, \tau)}
$$

The inequality in (31) is analogous to the condition that Williams (1999) uses in the trading model with an arbitrary number of sellers and buyers to determine whether the efficient trading rule can be implemented. To push the analogy further, we can exploit the fact that we have transformed our interdependent values model into a private values model to provide the following two corollaries, which establish the formal equivalence of our findings to the analysis of Myerson and Satterthwaite (1983) and Williams (1999).

Corollary 1 Assume that $n=1$. If $\underline{v}+\underline{w}<0, p^{*}$ is not implementable.

This result is equivalent to the impossibility result of Myerson and Satterthwaite (1983). Indeed, for any realization of $(\sigma, \tau)$, we can define $c:=\delta-w$ and $b:=\delta+v$ for any $\delta>\bar{w}_{1}$, and reinterpret the model as follows: agent 0 is a seller with cost $c$ and agent 1 is a buyer with value $b$. The gains from trade are $b-c=v+w$, and the condition $\underline{v}+\underline{w}<0$ is equivalent to $\underline{b}-\bar{c}<0$, which is the 
'no gap' condition of overlapping supports in Myerson and Satterthwaite. ${ }^{15}$

We can extend the analogy to the private values trading model. The case with $n$ bidders in our interdependent values merger model is analogous to the case with one seller and $n$ buyers in the trading model; therefore, the analysis of Williams (1999) applies.

Corollary 2 Suppose all synergy values are drawn fron the same distribution, with $\left[\underline{v}_{i}, \bar{v}_{i}\right]=$ $\left[\underline{w}_{i}, \bar{w}_{i}\right]=[l, h] ;$ and let $\left[\underline{v}_{i}^{(\sigma, \tau)}, \bar{v}_{i}^{(\sigma, \tau)}\right]$ and $\left[\underline{w}_{i}^{(\sigma, \tau)}, \bar{w}_{i}^{(\sigma, \tau)}\right]$ denote the supports of $v_{i}$ and $w_{i}$, for any realization of $(\sigma, \tau)$. If, for some $\sigma$ and $\tau$, we have $\left[\underline{v}_{i}^{(\sigma, \tau)}, \bar{v}_{i}^{(\sigma, \tau)}\right]=\left[\underline{w}_{i}^{(\sigma, \tau)}, \bar{w}_{i}^{(\sigma, \tau)}\right]=[l, h]$, $i=1, \ldots, n$, and $l+h \leq 0$, then $p^{*}$ is not implementable.

The inequality $l+h \leq 0$ implies that no merger takes place when the target observes the lowest realizations of the synergy values compatible with $(\sigma, \tau)$. Corollary 2 tells us that it is impossible to implement an efficient merger rule unless we can guarantee that the synergies from the worst possible merger outcome are strictly positive. In other words, whenever no bidder can be certain that a proposed merger is value-enhancing, the efficient merger rule $p^{*}$ is not implementable.

What happens if the inequality in Corollary 2 is violated for all realizations of $(\sigma, \tau)$ ? We cannot conclude that $p^{*}$ is implementable. To see this, simply observe the previous inequality is compatible with the 'no gap' condition in Corollary 1. However, as we describe in the following remark, one can establish a positive result along the lines of cases 2 and 3 in Table I, following Theorem 4, in Williams (1999):

Remark 1 Suppose that $\max _{i \in N}\left[\underline{w}_{i}^{(\sigma, \tau)}+\bar{v}_{i}^{(\sigma, \tau)}\right]>0$ for all realizations of $(\sigma, \tau)$. Then, if $n$ is sufficiently large, the efficient merger rule $p^{*}$ is implementable.

The proof of this is a straightforward extension of the proof of Theorem 4 in Williams (1999): replacing heterogeneous $n>1$ with $n$ identical realizations of the worst type immediately yields

\footnotetext{
${ }^{15}$ We are grateful to a referee for suggesting this interpretation.
} 
the result. We omit the full proof for the sake of brevity.

These results highlight the fact that despite multiple means of payment and ex post verifiability we are still in a world in which private information has a significant impact on merger outcomes. Double-sided asymmetric information is problematic for mergers, unless the degree of competition is large, even when transfers can be based on ex post observable information. It is not necessary to resort to hubris, private benefits, entrenchment, or other agency costs to take a dim view of the efficiency of mergers: inefficiencies may well be present even when all actors are maximizing firm value.

We close this section with an observation about the two-pronged nature of these inefficiencies. First, asymmetric information causes too few firms to merge; i.e., when condition (29) is violated, gains from trade go unexploited with positive probability. Moreover, when there is more than one bidder, in the second best outcome it is often the case that the 'wrong' merger occurs. That is, the second best outcome generically involves the target merging with a bidder who offers less than maximal synergies.

In the trading model, this second type of error does not occur if the $n$ buyers are ex ante identical. In our merger model, however, even an ex ante symmetric model will result in an ex post asymmetric realization of $\sigma$ and $\tau$, inducing suboptimal mergers in almost all cases.

\section{Mergers with Unverifiable Firm Values}

In this section we remove the assumption that transfers can be conditioned on ex post observable information. The crucial implication of this is that stock and cash are no longer equivalent methods of payment, and any stock transfer will necessarily generate information rents which hinder the implementation of efficient merger rules.

The information structure analyzed in the previous section allowed us to collapse private in- 
formation about stand-alone values and synergies into a single-dimensional variable. This is no longer possible when the ex post value of the merged firm is unverifiable. Now what matters a great deal for the efficiency merger outcomes is whether an agent's private information concerns its stand-alone values or its potential synergies.

In this setting, acquisition mechanisms emerge as the best feasible mechanisms in a wide range of situations. In an acquisition mechanism, one party completely buys out the other party, leaving them with zero ownership in the firm going forward.

Our analysis demonstrates the general principle that hidden information about stand-alone values is more problematic for merger efficiency that hidden information about synergies. In one extreme, when all stand-alone values are known, private information about potential synergies poses no difficulty in the implementation of efficient merger rules. In fact, efficient merger rules can always be implemented, i.e., there is no wedge between first and second best, even without cash transfers.

In the opposite extreme, when each firm's stand-alone value is privately known, and there is no uncertainty about the (positive) synergy values, the implementation of efficient rules requires the synergy to be sufficiently large. This is due to the adverse selection effect that we described in the introduction: willingness to merge conveys bad news about stand-alone values and thus has a negative impact on the willingness to merge by a potential partner.

These extreme situations illustrate a more general phenomenon which helps us to answer the question of who should buy whom. Our analysis establishes that, in general, the party with the greatest informational rents should be the acquirer, and should own the firm ex post. In general, whenever most of the uncertainty in value arises from synergies, then the party with the larger contribution to synergies should own the firm outright. Conversely, the party with the larger stand-alone value should own the firm ex post when most of the uncertainty surrounds stand-alone 
values.

The results in this section are similar to the analysis of partnerships in Fieseler, Kittsteiner and Moldovanu (2003), in which eagerness to sell one's share of the partnership is bad news for the other agent when one's valuation depends positively on one's signal, but is good news when one's valuation depends negatively on one's signal. In the remainder of the section we turn first to the case of known stand-alone values and a single bidder. Then we analyze the case of a single bidder with known synergies. We conclude by offering some empirical observations based on this analysis.

\subsection{Known Stand-Alone Values}

In this section we show that, if all stand-alone values $x_{0}, x_{1}, \ldots, x_{n}$ are known, the efficient merger rule can always be implemented, without monetary transfers. ${ }^{16}$ In other words, there exists a mechanism $\left(p^{*}, s, 0\right)$ that is ex post budget-balanced, incentive compatible and individually rational.

The mechanism $\left(p^{*}, s, 0\right)$ can be described as follows. Define $X$ to be the sum of stand-alone values across the target and all $n$ bidders and notice that, since we have assumed $\underline{x}_{i}>0$ each $i \in N_{0}$, we have $X>0$. Also define $V$ and $W$ to be the sets of all possible realizations of $v$ and $w$; formally, $X:=\sum_{j=0}^{n} x_{j}, V:=\times_{i \in N}\left[\underline{v}_{i}, \bar{v}_{i}\right]$ and $W:=\times_{i \in N}\left[\underline{w}_{i}, \bar{w}_{i}\right]$.

If, according to the agents' reports, no merger generates positive synergies, then nothing happens, i.e. each owner retains full ownership of his or her firm. Otherwise, the merger generating the highest synergy takes place, and agent $j$ receives fraction $\frac{x_{j}}{X}$ of each non-merging firm, as well as of the new firm. Formally, for all $(v, w)$ and all firms $j$,

$$
s_{j j}^{0}(v, w)=1, \text { and } s_{k j}^{i}(v, w)=\frac{x_{j}}{X}, \text { for all bidders } i, \text { all firms } k .
$$

\footnotetext{
${ }^{16}$ This result generalizes immediately to the case in which $x_{i}$ is $i$ 's private information, but it is sufficiently costly for $i$ to report $\widehat{x}_{i}>x_{i}$, while reporting $\widehat{x}_{i} \leq x_{i}$ can be costless. This may be the case when $x_{i}$ is given by tangible assets and it is costly to engage in fraudulent overvaluation of the assets.
} 
In the game induced by the mechanism $\left(p^{*}, s, 0\right)$, agent $j$ 's ex post expected surplus when the true type profile is $(v, w)$ and the agents report $(\widehat{v}, \widehat{w})$ is

$$
\begin{aligned}
\widehat{u}_{j}(\widehat{v}, \widehat{w}, v, w) & =p_{0}^{*}(\widehat{v}, \widehat{w}) x_{j}+\sum_{i \in N} p_{i}^{*}(\widehat{v}, \widehat{w})\left[\frac{x_{j}}{X} \sum_{k \in N \backslash\{i\}} x_{k}+\frac{x_{j}}{X}\left(x_{0}+x_{i}+w_{i}+v_{i}\right)\right]-x_{j} \\
& =p_{0}^{*}(\widehat{v}, \widehat{w}) x_{j}+\sum_{i \in N} p_{i}^{*}(\widehat{v}, \widehat{w}) x_{j}+\sum_{i \in N} p_{i}^{*}(\widehat{v}, \widehat{w}) \frac{x_{j}}{X}\left(w_{i}+v_{i}\right)-x_{j} \\
& =\frac{x_{j}}{X} \sum_{i \in N} p_{i}^{*}(\widehat{v}, \widehat{w})\left(v_{i}+w_{i}\right) .
\end{aligned}
$$

It is immediate to see that truth-telling is a best response to truth-telling for all firms. Furthermore, in the truth-telling equilibrium individual rationality is always satisfied. At last, we observe that all firms are ex post happy to implement the stock swaps and the merger prescribed by the mechanism as long as the synergies from the proposed merger $v_{i}+w_{i}$ are positive. This is always the case on the equilibrium path, thus implying the the mechanism is ex post individually rational.

We have thus proved the following result.

Proposition 2 If the stand-alone values $x_{0}, x_{1}, \ldots, x_{n}$ are commonly known, the efficient merger rule $p^{*}$ is implementable with a mechanism that does not use monetary transfers and is ex post individually rational.

When there is only one potential partner the mechanism is particularly simple and realistic. If the two firms find out that the sum of the reported synergies is negative then nothing happens; otherwise the merger takes place and each agent $i=0,1$ receives fraction $\frac{x_{i}}{x_{0}+x_{1}}$ of the new firm. It is optimal to ignore the (claimed) synergy values, using instead only the verifiable stand-alone values.

When there are multiple firms, the problem becomes to select the merger producing the highest synergies. Here the difficulty is to make sure that potential partners do not overstate the value of 
their synergies in order to increase the probability of a merger with their own firm. The mechanism avoids this by giving to each potential partner a fraction of the synergies of the most successful merger. With multiple potential partners the mechanism is clearly less realistic. However, a similar result can be approximated when the group of owners engage in cross-selling of shares, each owner acquiring toeholds in other firms. ${ }^{17}$ The acquisition of toeholds tends to align the incentives of owners to efficiency, since now each owner benefits from any merger with positive synergy, and has an interest in making sure that the merger with the highest synergy occurs.

Many mergers are the result of negotiations involving only two firms, and are implemented via stock swaps only (i.e., no cash transfers). Proposition 2 suggest that such transaction may well be efficient if there is little uncertainty on the stand-alone values. It also shows why uncertainty on the synergies may not be a big problem, compared to uncertainty about stand alone values. When uncertainty is only about the synergies, we can always arrange the property structure in such a way that the owners retain their firm when no merger occurs, and get an equivalent value plus a share of the synergies when a merger occurs. This makes sure that the owners have a common interest in finding out the highest synergies. This does not happen when uncertainty is about the stand alone values, the next case we examine.

\subsection{Known Synergies with One Partner}

For simplicity, we focus attention to the case in which there is a single bidder. Suppose now that the stand-alone value of each firm is private information, but that the synergy values are known to be positive: that is, $\omega:=w_{1}+v_{1}>0$. The efficient merger rule in this case is $p_{1}^{*}\left(x_{0}, x_{1}\right)=1$ for each $\left(x_{0}, x_{1}\right)$. In any efficient mechanism agent $i$ 's ex post expected surplus when the true types

\footnotetext{
${ }^{17}$ We thank a referee for suggesting this interpretation.
} 
are $x=\left(x_{0}, x_{1}\right)$ and the reported ones are $x^{\prime}=\left(x_{0}^{\prime}, x_{1}^{\prime}\right)$, is given by

$$
\widehat{u}_{i}\left(x^{\prime}, x\right)=\left(x_{0}+x_{1}+\omega\right) s_{0 i}^{1}\left(x^{\prime}\right)+t_{i}^{1}\left(x^{\prime}\right)-x_{i}, \quad i=0,1 .
$$

To simplify the exposition, suppose also that $x_{0}$ and $x_{1}$ are identically distributed with support $[\underline{x}, \bar{x}]$, c.d.f. $\Phi$ and density $\phi .^{18}$ In this case, under a standard regularity condition on $\Phi$, we have the following necessary and sufficient condition for the implementation of $p^{*}$.

Proposition 3 Suppose that $\frac{\Phi(\cdot)}{\phi(\cdot)}$ is nondecreasing. Then the efficient merger rule $p^{*}$ can be implemented if and only if

$$
\int_{\underline{x}}^{\bar{x}}[1-\Phi(\psi)] \Phi(\psi) d \psi \leq \frac{\omega}{2} .
$$

The important implication of Proposition 3 is that, whenever $\omega$ is positive but smaller than the critical value $\omega_{*}:=2 \int_{\underline{x}}^{\bar{x}}[1-\Phi(\psi)] \Phi(\psi) d \psi$, no incentive-feasible mechanism can guarantee that the two firms merge with probability one, even if there is no uncertainty about the synergies being positive.

This result hinges on the fact that, since we have assumed that the value of the new firm is unverifiable, the agents' information rent now depend on both the merger rule $p$ and the sharing rule $s$. In the previous section ex post verifiability made the particular sharing rule immaterial. Since the value of the new firm is not verifiable ex post, the sharing rule has an impact on each firm's expected utility.

The proof of Proposition 3 consists of the following steps. We begin by pointing out that agent $i$ 's expected information rent is determined by the function $S_{i}\left(x_{i}\right) \equiv E_{x_{-i}}\left[s_{0 i}^{1}\left(x_{i}, x_{-i}\right)\right]$. That is, the standard characterization of incentive compatibility in this case yields the envelope condition

$$
U_{i}\left(x_{i}\right)=U_{i}(\bar{x})+\int_{x_{i}}^{\bar{x}}\left[1-S_{i}(s)\right] d s, \quad \text { for all } x_{i} \in[\underline{x}, \bar{x}] .
$$

\footnotetext{
${ }^{18}$ Both Propositions 3 and 4 readily generalize to the case where firms are drawn from different distributions. The main difference is that instead of a single cdf $\Phi$, the proofs involve two cdfs, $\Phi_{1}$ and $\Phi_{2}$.
} 
This, together with the observation that $1-S_{i}\left(x_{i}\right) \geq 0$ for all $x_{i} \in[\underline{x}, \bar{x}]$, in turn implies that in any incentive compatible mechanism individual rationality is satisfied if and only if $U_{i}(\bar{x}) \geq 0$. Thus the type of each agent who is the most reluctant to participate, i.e. who earns the lowest net surplus relative to his stand-alone value, is $\bar{x}$.

Taking the expectation with respect to $x_{i}$ on both sides in (33), integrating the last term by parts, summing across the two agents, and solving for $\sum_{i=0}^{1} U_{i}(\bar{x})$ yields

$$
\sum_{i=0}^{1} U_{i}(\bar{x})=\sum_{i=0}^{1} \int_{\underline{x}}^{\bar{x}} U_{i}\left(x_{i}\right) d \Phi\left(x_{i}\right)-\sum_{i=0}^{1} \int_{\underline{x}}^{\bar{x}} \Phi\left(x_{i}\right)\left[1-S_{i}\left(x_{i}\right)\right] d x_{i} .
$$

Since individual rationality implies $\sum_{i=0}^{1} U_{i}(\bar{x}) \geq 0$, and the ex ante expected social surplus is just the synergy value, i.e. $\sum_{i=0}^{1} \int_{\underline{x}}^{\bar{x}} U_{i}\left(x_{i}\right) d \Phi\left(x_{i}\right)=\omega$, we arrive at the inequality

$$
\sum_{i=0}^{1} \int_{\underline{x}}^{\bar{x}} \Phi\left(x_{i}\right) \quad\left[1-S_{i}\left(x_{i}\right)\right] d x_{i} \leq \omega .
$$

In words: the sum of all information rents generated by any incentive feasible mechanism $\left(p^{*}, s, t\right)$ cannot exceed the total surplus $\omega$ that the merger produces.

The second step consists in showing that, when the hazard rate $\frac{\Phi}{\phi}$ is nondecreasing, the sum of all information rents (the left-hand side of (34)) is minimized, within the set of all feasible sharing rules, by the 'acquisition' rule $s^{A}$ defined by

$$
s_{00}^{A}\left(x_{0}, x_{1}\right) \equiv 1-s_{01}^{A}\left(x_{1}, x_{0}\right) \equiv \begin{cases}1, & \text { if } x_{0}>x_{1}, \\ 0, & \text { if } x_{0}<x_{1} .\end{cases}
$$

Thus $p^{*}$ cannot be implemented at all if it cannot be implemented with a mechanism in which the owner of the firm with the highest stand-alone value buys out the other firm entirely. Substituting $s^{A}$ into the previous inequality yields (32). This completes the proof of the 'only if' part of Proposition 3. 
To establish the 'if' part, we show that $p^{*}$ can be implemented when (32) holds with the sharing rule $s^{A}$ and the transfer rule $t^{A}$ defined by

$$
t_{0}^{A}\left(x_{0}, x_{1}\right) \equiv-t_{1}^{A}\left(x_{1}, x_{0}\right) \equiv \begin{cases}-m\left(x_{1}\right)-\frac{\omega}{2}, & \text { if } x_{0}>x_{1}, \\ m\left(x_{0}\right)+\frac{\omega}{2}, & \text { if } x_{0}<x_{1},\end{cases}
$$

where

$$
m\left(x_{i}\right) \equiv \frac{\int_{x_{i}}^{\bar{x}}(1-\Phi(s))^{2} d s}{\left(1-\Phi\left(x_{i}\right)\right)^{2}}+x_{i}=E\left[\min \left\{x_{0}, x_{1}\right\} \mid x_{0}, x_{1}>x_{i}\right]
$$

Given the impossibility of implementing the efficient rule $p^{*}$ when the synergy value $\omega$ is small, it is natural to ask which mechanisms maximize the ex-ante expected joint surplus.

The acquisition mechanism defined in (36) is quite intuitive. Remember that the essential incentive problem is that firms with high stand-alone values are reluctant to sell. Thus the newly merged firm is sold to the owners claiming to have the highest stand-alone value, at a price that depends positively on the stand-alone value of the other firm (the term $\frac{\omega}{2}$ is added to share the surplus). This provides a countervail to the incentive to exaggerate the stand alone value, and a careful choice of the function $m\left(x_{i}\right)$ provides the correct incentives for truth-telling. Essentially in an acquisition mechanism the 'large' firm buys the 'small' firm, at a price that gives the small firm part of the surplus. This appears to be a common feature of many mergers.

Our last proposition establishes that acquisition mechanisms are optimal even when full efficiency is not attainable.

Proposition 4 Suppose that ex post efficiency is not attainable, and assume that $\frac{\Phi(\cdot)}{\phi(\cdot)}$ is nondecreasing. Then the joint expected surplus

$$
\max _{(p, s, t)} \sum_{i=0}^{1} \int_{\underline{x}}^{\bar{x}} U_{i}\left(x_{i}\right) d \Phi\left(x_{i}\right),
$$


is maximized, subject to incentive compatibility, individual rationality and ex post budget balance, by an acquisition mechanism $\left(p, s^{A}, t^{A}\right)$, where $s^{A}$ and $t^{A}$ are defined in (35) and (36), respectively.

The results of this section establish the identity of the bidder and target, as well as the optimality of cash acquisitions as a takeover mechanism. To answer the question who buys whom, this section shows that the firm with the highest stand-alone value should acquire the firm with the lower stand-alone value. To answer the question how should the deal be structured, this section shows that the acquirer should buy out the target shareholders with cash. ${ }^{19}$

\subsection{Discussion}

The preceding results have been couched in terms of two extremes: one in which stand-alone values are completely known; the other in which synergies are completely known. In fact, these are just the two ends of a continuum, and in general the larger take-away from this section is that whenever there is more uncertainty about stand-alone values, the firm with the larger stand-alone values should be the acquirer.

Does this tell us anything about empirical patterns in merger activity? Can we use these general findings to offer theoretical guidance about when we expect certain types of mergers to occur? Or, more generally, do our predictions square with existing empirical evidence on mergers and acquisitions? We end this section by discussing the empirical insights that arise from distinguishing uncertainty about stand-alone values from uncertainty about synergies. ${ }^{20}$

\footnotetext{
${ }^{19}$ In this paper we assume that the potential acquirer has always enough cash. Clearly, if the acquirer had to raise cash in the capital markets the kind of adverse selection problems discussed in Myers and Majluf (1984) would appear.

${ }^{20}$ Another approach would be to focus on empirical predictions that arise from the contrast of the verifiable values case (Section 3) with the unverifiable values case (Section 4). This requires a discussion of the market's expectations at the time of the merger announcement, which is beyond the scope of our analysis. However, in light of the role of
} 
The first insight concerns the frequency of merger activity. When most of the uncertainty surrounds synergies, we have shown that the efficient merger rule is generally easy to implement. Mergers are feasible even when the expected synergies are small. In contrast, when most of the uncertainty surrounds stand-alone values, it takes larger expected synergies to overcome information asymmetries.

This, in turn, implies that we should expect to see more mergers occurring when merger activity is being driven by synergy-related considerations. We should expect fewer mergers when the bulk of the uncertainty in the merger surrounds stand-alone values of existing assets. This squares with the empirical observation that productivity shocks in industries produce merger waves (see Gort (1969), Rhodes-Kropf, Robinson and Viswanathan (2005) and Harford (2005)), since it it is often argued that the productivity shocks create new ways of recombining existing assets to create valuable, but uncertain, synergies.

This interpretation also squares with the empirical evidence in Mitchell and Mulherin (1996) and Mulherin and Boone (2000) on industry clustering. These authors show that merger activity clusters at the industry level, and argue using event-study techniques that it is predominantly driven by the desire to realize synergies. They also show that spikes in industry activity follow industry deregulations. One interpretation of these findings in light of our results is that regulation allows stand-alone values to become more readily observable, but impedes the realization of synergies. When synergies arise, this creates a pressure to deregulate the industry, and in response to industry deregulation, a spate of mergers occurs.

The second insight concerns the role of liquidity constraints in shaping merger outcomes. In the case when the uncertainty mostly surrounds synergies, liquidity constraints should only affect the means of payment, not the amount of merger activity that should occur. This is because stock method of payment in increasing ex post verifiability, as discussed in Section 2, see Eckbo, Giammarino, and Heinkel (1990) for early evidence on how market reactions are related to the medium of exchange in takeovers. 
and cash are equally feasible means for acquiring a target firm when the bulk of the uncertainty surrounds synergies. On the other hand, liquidity constraints should also affect the amount of merger activity that occurs when the bulk of the uncertainty surrounds the value of stand-alone assets. This fits with the empirical observation that in times when merger activity is high, i.e., during merger waves, the amount of stock-financed merger activity spikes dramatically, because in our model cash is not necessary when only synergies are uncertain. It is also broadly consistent with the observation that when liquidity is tight, i.e., during economic downturns when most mergers involve industry consolidation, the acquisition market is often dominated by financial buyers who purchase assets at steep discounts.

In broad terms, the analysis of this section squares well with the some of the differences between expansionary and contractionary merger waves described in Andrade and Stafford (2004). Pushing these insights into full-blown empirical predictions is beyond the scope of this paper. In particular, we do not model liquidity constraints, and a more-detailed specification of what market participants know is required than our theory offers. But these insights nonetheless offer some tantalizing links to the data, which we hope can guide further empirical work.

\section{Conclusions}

This paper uses a mechanism design approach to study how private information impacts the overall efficiency of mergers and acquisitions. We study a very general and realistic situation in which a single target faces many potential bidders, with all parties holding private information about both their own stand-alone values as well as their contribution to the overall synergies of a potential transaction.

We focus on two cases: when the value of the new firm is verifiable ex post, and when it is not. In the first case, using shares of non-merging firms as means of payment generates information rents 
for the owners and thus hinders the implementation of efficient rules. Thus, the optimal merger rule never involves redistribution of failed bidders' shares. We identify a particular sharing rule that allows us to recast the inter-dependent values merger problem as a private value framework, allowing us to provide necessary and sufficient conditions for the implementation of efficient merger rules. Our main finding from this part of the analysis is bad news for merger efficiency: when all parties are always uncertain about the efficiency of merging or the number of firms is large, the first-best cannot can be implemented.

When ex post values cannot be verified, the results hinge critically on the nature of private information. Private information about synergy values never hinders the implementation of efficient merger rules, but asymmetric information about stand-alone values creates a gap between first and second best that cannot be overcome, even in cases where it is common knowledge that the merger synergies are always positive.

This difference can be understood intuitively as follows. In the case of unknown synergies, types who are more optimistic about the value of a new firm are also more eager to merge. Thus an agent's willingness to merge is good news for his or her potential partner, and this facilitates the design of efficient mechanisms. If instead there is private information about the firm's stand-alone values an agent's willingness to merge is bad news for his or her potential partner because types with lower values have less to gain from merging.

Our results show that asymmetric information alone can give rise to a theory of optimal ownership even when there are no issues with ex post control rights, no private benefits, and no agency costs. In the presence of private information about stand-alone values, the best possible merger outcome is a cash acquisition in which the firm with the greatest amount of uncertainty about stand-alone values buys the other firm. This is implementable as long as the expected synergies are sufficiently large. In contrast, when synergies are uncertain, mergers with stock are implementable even without cash, for any level of positive synergies, no matter how small. 
Therefore, our theory provides new insights into the patterns of merger activity that we observe across industries and over time. Our theory suggests that industry shocks in expansionary periods lead to merger waves because they give rise to new (hence relatively uncertain) synergies between firms. During these times, mergers are easy to implement and the method of payment becomes unimportant. This helps to explain the widespread use of stock as a means of payment during these episodes. In contrast, mergers that involve industry consolidation are less about exploiting uncertain synergies and more about reducing overcapacity; liquidity plays an important role in these mergers, which helps explain why financial buyers, instead of industry buyers, are relatively more common here. Building more detailed empirical predictions and tests from these insights is a promising task for future work.

Department of Economics, SUNY at Stony Brook, USA, and Departamento de Economia de la Empresa, Universidad Carlos III de Madrid, Spain (Brusco); Fuqua School of Business, Duke University, USA (Lopomo, Robinson, Viswanathan) 


\section{Appendix}

Proof of Lemma 1. Consider any feasible mechanism $(p, s, t)$ and any feasible sharing rule $\widetilde{s}$ that differs from $s$ only in the ownership structure of any new firm $\widetilde{s}_{0 .}^{i}=\left(\widetilde{s}_{00}^{i}, \widetilde{s}_{01}^{i}, \ldots, \widetilde{s}_{0 n}^{i}\right), i \in N$. Define $\tilde{t}$ as

$$
\widetilde{t}_{j}^{i}(\theta) \equiv t_{j}^{i}(\theta)+r_{i}\left[s_{0 j}^{i}(\theta)-\widetilde{s}_{0 j}^{i}(\theta)\right]
$$

Note that the new transfer rule $\widetilde{t}$ depends on the verifiable values $r_{1}, \ldots, r_{n}$.

Since $s$ and $\widetilde{s}$ are both feasible, we have $\sum_{j \in N_{0}} s_{0 j}^{i}(\theta)=\sum_{j \in N_{0}} \widetilde{s}_{0 j}^{i}(\theta)=1$, hence

$$
\sum_{j \in N_{0}} \widetilde{t}_{j}^{i}(\theta)=\sum_{j \in N_{0}} t_{j}^{i}(\theta)=0, \text { for all } \theta \in \Theta, i \in N
$$

Thus $\tilde{t}$ is budget balanced.

Now let $\widehat{u}_{j}\left(\theta^{\prime}, \theta \mid i\right)$ and $\widetilde{u}_{j}\left(\theta^{\prime}, \theta \mid i\right)$ denote agent $j$ 's surplus, conditional on firm 0 and $i$ merging (or no merger, for $i=0)$ in the revelation game induced by $(p, s, t)$ and $(p, \widetilde{s}, \widetilde{t}$ ) respectively, when the agents' types are $\theta$ and they report $\theta^{\prime}$. Since $s^{0}=\widetilde{s}^{0}$, we have $\widehat{u}_{j}\left(\theta^{\prime}, \theta \mid 0\right)=\widetilde{u}_{j}\left(\theta^{\prime}, \theta \mid 0\right)$. Furthermore, for each $i \in N$, we have

$$
\begin{aligned}
\widetilde{u}_{j}\left(\theta^{\prime}, \theta \mid i\right) & =r_{i} \widetilde{s}_{0 j}^{i}\left(\theta^{\prime}\right)+\sum_{k \in N \backslash\{i\}} s_{k j}^{i}\left(\theta^{\prime}\right) x_{k}+\widetilde{t}_{j}^{i}\left(\theta^{\prime}\right)-x_{j} \\
& =r_{i} \widetilde{s}_{0 j}^{i}\left(\theta^{\prime}\right)+\sum_{k \in N \backslash\{i\}} s_{k j}^{i}\left(\theta^{\prime}\right) x_{k}+t_{j}^{i}\left(\theta^{\prime}\right)+r_{i}\left[s_{0 j}^{i}\left(\theta^{\prime}\right)-\widetilde{s}_{0 j}^{i}\left(\theta^{\prime}\right)\right]-x_{j} \\
& =r_{i} s_{0 j}^{i}\left(\theta^{\prime}\right)+\sum_{k \in N \backslash\{i\}} s_{k j}^{i}\left(\theta^{\prime}\right) x_{k}+t_{j}^{i}(\theta)-x_{j} \\
& =\widehat{u}_{j}\left(\theta^{\prime}, \theta \mid i\right) .
\end{aligned}
$$

Therefore

$$
\widetilde{u}_{j}\left(\theta^{\prime}, \theta\right)=\sum_{i \in N_{0}} p_{i}\left(\theta^{\prime}\right) \widetilde{u}_{j}\left(\theta^{\prime}, \theta \mid i\right)=\sum_{i \in N_{0}} p_{i}\left(\theta^{\prime}\right) \widehat{u}_{j}\left(\theta^{\prime}, \theta \mid i\right)=\widehat{u}_{j}\left(\theta^{\prime}, \theta\right)
$$


Thus agent $j$ 's ex post expected surplus functions $\widehat{u}_{j}\left(\theta^{\prime}, \theta\right)$ and $\widetilde{u}_{j}\left(\theta^{\prime}, \theta\right)$, in the revelation game induced by $(p, s, t)$ and $(p, \widetilde{s}, \widetilde{t})$ respectively, are the same.

Proof of Lemma 2. For any $\sigma \in \times_{i \in N}\left[\underline{x}_{0}+\underline{w}_{i}, \bar{x}_{0}+\bar{w}_{i}\right]$, define $C_{0}^{\sigma}(\zeta) \equiv U_{0}^{\sigma}(\zeta)+\zeta B_{0}^{\sigma}(\zeta)$, $\zeta \in\left[\underline{x}^{\sigma}, \bar{x}^{\sigma}\right]$. The inequalities in (11) imply

$$
\begin{aligned}
U_{0}^{\sigma}(\zeta) & \geq C_{0}^{\sigma}\left(\zeta^{\prime}\right)-\zeta B_{0}^{\sigma}\left(\zeta^{\prime}\right) \\
& =U_{0}^{\sigma}\left(\zeta^{\prime}\right)+\left(\zeta^{\prime}-\zeta\right) B_{0}^{\sigma}\left(\zeta^{\prime}\right), \quad \zeta, \zeta^{\prime} \in\left[\underline{x}^{\sigma}, \bar{x}^{\sigma}\right],
\end{aligned}
$$

which in turn implies

$$
\left(\zeta^{\prime}-\zeta\right) B_{0}^{\sigma}(\zeta) \geq U_{0}^{\sigma}(\zeta)-U_{0}^{\sigma}\left(\zeta^{\prime}\right) \geq\left(\zeta^{\prime}-\zeta\right) B_{0}^{\sigma}\left(\zeta^{\prime}\right), \quad \zeta, \zeta^{\prime} \in\left[\underline{x}^{\sigma}, \bar{x}^{\sigma}\right]
$$

The two inequalities in (38) immediately imply that $B_{0}^{\sigma}$ is non-increasing, hence continuous almost everywhere. Taking $\zeta^{\prime}>\zeta$, dividing by $\zeta^{\prime}-\zeta$, and letting $\zeta^{\prime} \rightarrow \zeta$ yields

$$
\frac{d U_{0}^{\sigma}(\zeta)}{d \zeta}=-B_{0}^{\sigma}(\zeta) \text { a.e. }
$$

Since $B_{0}^{\sigma}$ is bounded, (38) also implies that $U_{0}^{\sigma}$ is Lipschitz, hence absolutely continuous. Therefore we can integrate both sides in (39) and obtain (17). The proof of the second half of the lemma is analogous.

Proof of Lemma 3. Suppose that $(p, s, t)$ is incentive-feasible, satisfies weak budget balance, and without loss of generality (by Lemma 1 ) suppose that $s_{00}^{i}(\theta)=1$ for all $\theta \in \Theta, i \in N$. Consider a new mechanism $(p, \widetilde{s}, \widetilde{t})$ where: 
1. the sharing rule $\widetilde{s}$ is determined by the LU property, defined in (7), and by the fact that agent 0 becomes the sole owner of any new firm, $\widetilde{s}_{00}^{i}(\theta)=1$ for all $\theta \in \Theta, i \in N$;

2. the transfer rule $\widetilde{t}$ is designed so that the change in the total value received (both in shares and cash) by each type of each agent, for each realization of her opponents' types, leaves the lowest information rent which is consistent with the incentive compatibility and individual rationality constraints.

More precisely, for each $i \in N$ the transfer functions are defined as follows:

$$
\begin{aligned}
& \widetilde{t}_{i}^{0}(\theta)=t_{i}^{0}(\theta)+\sum_{k \in N_{0} \backslash\{i\}} s_{k i}^{0}(\theta) x_{k}-\left[1-s_{i i}^{0}(\theta)\right] x_{i}-\int_{x_{i}}^{\bar{x}_{i}^{\tau_{i}}} A_{i}^{\tau_{i}}(\zeta) d \zeta-u_{i}\left(\left(\bar{x}_{i}^{\tau_{i}}, \tau_{i}\right), \theta_{-i}\right), \\
& \widetilde{t}_{i}^{j}(\theta)=t_{i}^{j}(\theta)+\sum_{k \in N \backslash\{i, j\}} s_{k i}^{j}(\theta) x_{k}-\left[1-s_{i i}^{j}(\theta)\right] x_{i}-\int_{x_{i}}^{\tau_{i}^{i}} A_{i}^{\tau_{i}}(\zeta) d \zeta-u_{i}\left(\left(\bar{x}_{i}^{\tau_{i}}, \tau_{i}\right), \theta_{-i}\right), \quad j \in N \backslash\{i\}, \\
& \widetilde{t}_{i}^{i}(\theta)=t_{i}^{i}(\theta)+\sum_{k \in N \backslash\{i\}} s_{k i}^{i}(\theta) x_{k}-\int_{x_{i}}^{\tau_{i}^{\tau_{i}}} A_{i}^{\tau_{i}}(\zeta) d \zeta-u_{i}\left(\left(\bar{x}_{i}^{\tau_{i}}, \tau_{i}\right), \theta_{-i}\right),
\end{aligned}
$$

where

$$
A_{i}^{\tau_{i}}(\zeta) \equiv B_{i}^{\tau_{i}}(\zeta)-P_{i}\left(\zeta, \tau_{i}\right), \quad \zeta \in\left[\underline{x}_{i}^{\tau_{i}}, \bar{x}_{i}^{\tau_{i}}\right]
$$

For agent 0 transfers are given by

$$
\begin{aligned}
& \widetilde{t}_{0}^{0}(\theta)=t_{0}^{0}(\theta)+\sum_{k \in N} s_{k 0}^{0}(\theta) x_{k}-\left[1-s_{00}^{0}(\theta)\right] x_{0}-\int_{x_{0}}^{\bar{x}_{0}^{\sigma}} A_{0}^{\sigma}(\zeta) d \zeta-u_{0}\left(\left(\bar{x}_{0}^{\sigma}, \sigma\right), \theta_{-i}\right), \\
& \widetilde{t}_{0}^{i}(\theta)=t_{0}^{i}(\theta)+\sum_{k \in N \backslash\{i\}} s_{k 0}^{i}(\theta) x_{k}-\int_{x_{0}}^{\bar{x}_{0}^{\sigma}} A_{0}^{\sigma}(\zeta) d \zeta-u_{0}\left(\left(\bar{x}_{0}^{\sigma}, \sigma\right), \theta_{-i}\right),
\end{aligned}
$$

where

$$
A_{0}^{\sigma}(\zeta) \equiv B_{0}^{\sigma}(\zeta)-\left[1-P_{0}(\zeta, \sigma)\right], \quad \zeta \in\left[\underline{x}_{0}^{\sigma}, \bar{x}_{0}^{\sigma}\right]
$$

It can be checked that the transfer rule $\widetilde{t}$ satisfies weak budget balance, i.e.

$$
\sum_{i \in N_{0}} \widetilde{t}_{i}^{j}(\theta) \leq 0, \quad \theta \in \Theta, \quad j \in N_{0}
$$


because the original transfer rule $t$ does, all shares of all firms sum up to one, and all functions $A_{0}^{\sigma}(\cdot)$ and $A_{i}^{\tau_{i}}(\cdot)$ take non-negative values for all $\sigma, \tau_{i}$, and $i \in N$.

Denoting the payoff functions in the initial mechanism as $u_{j}\left(\theta^{\prime}, \theta\right)$, and in the new mechanism as $u_{j}^{*}\left(\theta^{\prime}, \theta\right)$. We have

$$
u_{0}^{*}\left(\theta^{\prime}, \theta\right)=u_{0}\left(\theta^{\prime}, \theta\right)-\int_{x_{0}^{\prime}}^{\bar{x}_{0}^{\sigma}} A_{0}^{\sigma}(\zeta) d \zeta-u_{0}\left(\left(\bar{x}_{0}^{\sigma}, \sigma\right), \theta_{-i}\right)
$$

hence

$$
U_{0}^{*}\left(\theta_{0}^{\prime}, \theta_{0}\right)=U_{0}\left(\theta_{0}^{\prime}, \theta_{0}\right)-\int_{x_{0}^{\prime}}^{\bar{x}_{0}^{\sigma}} A_{0}^{\sigma}(\zeta) d \zeta-U_{0}\left(\left(\bar{x}_{0}^{\sigma}, \sigma\right)\right)
$$

and

$$
\begin{aligned}
U_{0}^{*}\left(\theta_{0}\right) & =U_{0}\left(\theta_{0}\right)-\int_{x_{0}^{\prime}}^{\bar{x}_{0}^{\sigma}} A_{0}^{\sigma}(\zeta) d \zeta-U_{0}\left(\left(\bar{x}_{0}^{\sigma}, \sigma\right)\right) \\
& =\int_{x_{0}^{\prime}}^{\bar{x}_{0}^{\sigma}} B_{0}^{\sigma}(\zeta) d \zeta-\int_{x_{0}^{\prime}}^{\bar{x}_{0}^{\sigma}} A_{0}^{\sigma}(\zeta) d \zeta \\
& =\int_{x_{0}^{\prime}}^{\bar{x}_{0}^{\sigma}} P_{0}(\zeta, \sigma) d \zeta \geq 0 .
\end{aligned}
$$

By the last inequality IR holds; and by the equality in (40) IC holds, because the original mechanism is incentive compatible. The proof that IC and IR hold for each agent $i \in N$ is analogous.

Proof of Proposition 1. Lemma 3 implies that, when we require only ex ante budget balance, we can restrict attention to mechanism that satisfy the LU property. Furthermore, by setting (without loss of generality) the sharing rules for the merged firm as in the text, the problem becomes a standard private value problem. The proof now simply adapts the results of Williams (1999) to our setting to show that condition (29) is necessary and sufficient for the existence of a Vickrey-ClarkeGroves (VCG) mechanism which satisfies ex ante budget balance. The result then follows from Proposition 5.6 in Krishna (2002) which states that a VCG mechanism satisfying ex ante budget 
balance exists if and only if there exists an efficient, incentive compatible and individually rational mechanism that satisfies ex post budget balance.

In any Groves-Clarke mechanism truth-telling is a dominant strategy and the payments are given by

$$
t_{i}\left(\theta_{i}, \theta_{-i}\right)=\sum_{j \neq i} \widetilde{v}_{j}\left(a^{*}\left(\theta_{i}, \theta_{-i}\right), \theta_{j}\right)-k_{i}
$$

where $k_{i}$ is a constant. The only issue is whether we can find constants $k_{i}$ for which individual rationality and ex ante budget balance are both satisfied. Since the expected sum of all transfers is

$$
E\left[\sum_{j \in N_{0}} t_{j}(\theta)\right]=E\left[\sum_{j \in N_{0}} \sum_{i \neq j} \widetilde{v}_{i}\left(a^{*}(\theta), \theta_{i}\right)-\sum_{j \in N_{0}} k_{j}\right]=n E\left[\sum_{i \in N_{0}} \widetilde{v}_{i}\left(a^{*}(\theta), \theta_{i}\right)\right]-\sum_{i \in N_{0}} k_{i},
$$

ex ante budget balance requires

$$
n E\left(\sum_{i \in N_{0}} \widetilde{v}_{i}\left(a^{*}(\theta), \theta_{i}\right)\right)=\sum_{i \in N_{0}} k_{i}
$$

individual rationality requires $\underline{Z}_{j}^{(\sigma, \tau)}-k_{j} \geq 0$ for all $j \in N_{0}$, hence

$$
\sum_{i \in N_{0}} k_{i} \leq \sum_{i \in N_{0}} \underline{Z}_{j}^{(\sigma, \tau)}
$$

Combining the two inequalities yields the condition

$$
n E\left(\sum_{i \in N_{0}} \widetilde{v}_{i}\left(a^{*}(\theta), \theta_{i}\right)\right) \leq \sum_{i \in N_{0}} \underline{Z}_{i}^{(\sigma, \tau)} .
$$

To see that this is also sufficient, note that the Groves-Clarke mechanism where $k_{i}=\underline{Z}_{i}^{(\sigma, \tau)}$ implements the efficient mechanism.

Proof of Corollary 1. When $n=1$ condition (29) becomes

$$
E_{v, w}^{\sigma, \tau}[\max \{v+w, 0\}] \leq E_{v}^{\sigma, \tau}[\max \{v+\underline{w}, 0\}]+E_{w}^{\sigma, \tau}[\max \{\underline{v}+w, 0\}], \forall \sigma, \tau,
$$


where $E_{v, w}^{\sigma, \tau}$ is the expectation taken over $(v, w)$ conditional on $(\sigma, \tau)$. This can be rewritten as

$$
E_{v, w}^{\sigma, \tau}[\max \{v+w, 0\}] \leq E_{v, w}^{\sigma, \tau}(\max \{(\underline{w}+v), 0\}+\max \{(w+\underline{v}), 0\}), \forall \sigma, \tau
$$

In particular, this must hold for $w^{\sigma, \tau}=\underline{w}$ and $v^{\sigma, \tau}=\underline{v}$. We claim that for each pair $(w, v)$ we have

$$
\max \{(w+v), 0\} \geq \max \{(\underline{w}+v), 0\}+\max \{(w+\underline{v}), 0\},
$$

with strict inequality for some $v$, thus implying that inequality (41) cannot be satisfied. Inequality (42) clearly holds if at least one term on the right hand side is zero. If they are both positive then the inequality becomes

$$
w+v \geq v+w+\underline{v}+\underline{w}
$$

which holds strictly because $\underline{v}+\underline{w}<0$.

Proof of Corollary 2. The proof of Corollary 2 hinges on the fact that since $l+h \leq 0$, no merger takes place if $\theta_{0}$ is such that $\underline{w}_{i}^{(\sigma, \tau)}=l$, for all $i \in N$, in which case $\underline{Z}_{0}^{(\tau, \tau)}=0$. On the other hand, if $v_{i}=l$ then there is no chance of a merger with firm $i$, and the expected social surplus is the expected value of a merger for the remaining $n-1$ firms. Let $G(\cdot)$ denote the c.d.f. of any sum $v_{i}+w_{i}$. Then, the c.d.f. of $\max _{j \in N \backslash i}\left\{v_{j}+w_{j}\right\}$ is $G^{n-1}(\cdot)$, and the expected social surplus when $v_{i}=\ell$ is

$$
\underline{Z}_{i}=\int_{0}^{2 h} z d G^{n-1}(z)
$$

for each $i$, so that

$$
\sum_{j \in N_{0}} \underline{Z}_{j}=\underline{Z}_{0}+\sum_{j \in N} \underline{Z}_{j}=n \int_{0}^{2 h} z d G^{n-1}(z) .
$$

On the other hand, the c.d.f. of $\max _{j \in N}\left\{v_{j}+w_{j}\right\}$ is $G^{n}(\cdot)$, so that the expected social surplus is

$$
E_{\theta}\left[\sum_{i \in N_{0}} \widetilde{v}_{i}\left(a^{*}(\theta), \theta_{i}\right)\right]=\int_{0}^{2 h} z d G^{n}(z)
$$

Thus in this case condition (29) becomes

$$
n \int_{0}^{2 h} z d G^{n}(z) \leq n \int_{0}^{2 h} z d G^{n-1}(z)
$$


which can never be satisfied, since the distribution on left-hand side stochastically dominates the one on the right. While the above argument holds for a specific set of realizations of $\sigma$ and $\tau$, the inequality in condition (43) will be satisfied for a positive measure set of realizations of $\sigma$ and $\tau$. It is worth observing explicitly that the impossibility result holds for any number of potential partners.

Proof of Proposition 3. We first show that the condition is necessary. The first step is to show that a mechanism $\left(p^{*}, s, t\right)$ satisfies both incentive compatibility and individual rationality only if

$$
\sum_{i=0}^{1} \int_{\underline{x}}^{\bar{x}} \Phi\left(x_{i}\right) \quad\left[1-S_{i}\left(x_{i}\right)\right] d x_{i} \leq \omega
$$

where $S_{i}\left(x_{i}\right) \equiv E_{x_{-i}}\left[s_{0 i}^{1}\left(x_{i}, x_{-i}\right)\right]$.

Fix a mechanism $\left(p^{*}, s, t\right)$. The interim expected surplus from reporting $x_{i}^{\prime}$, when the true stand-alone value is $x_{i}$, and the other agent reports $x_{-i}$ truthfully, can be written as

$$
\widehat{U}_{i}\left(x_{i}^{\prime} ; x_{i}\right)=E_{x_{-i}}\left[t_{i}^{1}\left(x_{i}^{\prime}, x_{-i}\right)+\left(\omega+x_{-i}\right) s_{0 i}^{1}\left(x_{i}^{\prime}, x_{-i}\right)\right]-\left[1-S_{i}\left(x_{i}^{\prime}\right)\right] x_{i}
$$

By standard arguments in mechanism design, as the ones used in Lemma 2, we have that $\left(p^{*}, s, t\right)$ satisfies incentive compatibility if and only if

1. the function $S_{i}$ is nondecreasing;

2. the function $U_{i}\left(x_{i}\right) \equiv \widehat{U}_{i}\left(x_{i}, x_{i}\right)$ satisfies the envelope condition

$$
U_{i}\left(x_{i}\right)=U_{i}(\bar{x})+\int_{x_{i}}^{\bar{x}}\left[1-S_{i}(y)\right] d y, \quad \text { for all } x_{i} \in[\underline{x}, \bar{x}]
$$

Taking expectations of both sides in (45), integrating by parts on the right-hand side, solving for $U_{i}\left(\bar{x}_{i}\right)$, and summing across the two agents yields

$$
\sum_{i=0}^{1} U_{i}\left(\bar{x}_{i}\right)=\sum_{i=0}^{1} \int_{\underline{x}_{i}}^{\bar{x}_{i}} U_{i}\left(x_{i}\right) d \Phi\left(x_{i}\right)-\sum_{i=0}^{1} \int_{\underline{x}_{i}}^{\bar{x}_{i}} \Phi\left(x_{i}\right)\left[1-S_{i}\left(x_{i}\right)\right] d x_{i} .
$$


Moreover, since the shares must sum up to one and the monetary transfers to zero, we have

$$
\sum_{i=0}^{1} \widehat{u}_{i}\left(x^{\prime}, x\right)=\left(x_{0}+x_{1}+\omega\right) \sum_{i=0}^{1} s_{0 i}^{1}\left(x^{\prime}\right)+\sum_{i=0}^{1} t_{i}^{1}\left(x^{\prime}\right)-\sum_{i=0}^{1} x_{i}=\omega
$$

hence $\sum_{i=0}^{1} \int_{\underline{x}}^{\bar{x}} U_{i}\left(x_{i}\right) d \Phi_{i}\left(x_{i}\right)=\omega$. Substituting this into (46), we obtain

$$
\sum_{i=0}^{1} U_{i}\left(\bar{x}_{i}\right)=\omega-\sum_{i=0}^{1} \int_{\underline{x}}^{\bar{x}} \Phi\left(x_{i}\right)\left[1-S_{i}\left(x_{i}\right)\right] d x_{i} .
$$

Finally, individual rationality requires $0 \leq \sum_{i=0}^{1} U_{i}\left(\bar{x}_{i}\right)$. Thus $\left(p^{*}, s, t\right)$ satisfies both incentive compatibility and individual rationality only if the condition in (44) holds.

The second step is to show that when the ratio $\frac{\Phi(\cdot)}{\phi(\cdot)}$ is nondecreasing, the sum of all information rents is minimized by the following sharing rule ${ }^{21}$

$$
s_{i}^{A}\left(x_{i}, x_{-i}\right)= \begin{cases}1, & \text { if } x_{i}>x_{-i}, \\ 0, & \text { if } x_{i}<x_{-i} .\end{cases}
$$

To prove this, write the information rents as

$$
\begin{gathered}
\sum_{i=0}^{1} \int_{\underline{x}}^{\bar{x}}\left[1-S_{i}\left(x_{i}\right)\right] \Phi\left(x_{i}\right) d x_{i}= \\
=\int_{\underline{x}}^{\bar{x}} \Phi\left(x_{0}\right) d x_{0}-\int_{\underline{x}}^{\bar{x}} S_{0}\left(x_{0}\right) \Phi\left(x_{0}\right) d x_{0}+\int_{\underline{x}}^{\bar{x}} \int_{\underline{x}}^{\bar{x}}\left[1-s_{01}^{1}\left(x_{1}, x_{0}\right)\right] d \Phi\left(x_{0}\right) \Phi\left(x_{1}\right) d x_{1} \\
=\int_{\underline{x}}^{\bar{x}} \Phi\left(x_{0}\right) d x_{0}-\int_{\underline{x}}^{\bar{x}} s_{00}^{1}\left(x_{0}, x_{1}\right) \frac{\Phi\left(x_{0}\right)}{\phi\left(x_{0}\right)} d \Phi\left(x_{0}\right) d \Phi\left(x_{1}\right)+\int_{\underline{x}}^{\bar{x}} \int_{\underline{x}}^{\bar{x}} s_{00}^{1}\left(x_{0}, x_{1}\right) \frac{\Phi\left(x_{1}\right)}{\phi\left(x_{1}\right)} d \Phi\left(x_{0}\right) d \Phi\left(x_{1}\right) \\
=\int_{\underline{x}}^{\bar{x}} \Phi\left(x_{0}\right) d x_{0}+\int_{\underline{x}}^{\bar{x}} \int_{\underline{x}}^{\bar{x}} s_{00}^{1}\left(x_{0}, x_{1}\right)\left[\frac{\Phi\left(x_{1}\right)}{\phi\left(x_{1}\right)}-\frac{\Phi\left(x_{0}\right)}{\phi\left(x_{0}\right)}\right] d \Phi\left(x_{0}\right) d \Phi\left(x_{1}\right) .
\end{gathered}
$$

If $\frac{\Phi(x)}{\phi(x)}$ is non-decreasing, the expression is minimized by setting $s_{00}^{1}\left(x_{0}, x_{1}\right)=0$ when $x_{1}>x_{0}$, and equal to 1 otherwise. This is exactly what the acquisition mechanism does. Substituting $s^{A}$ into (44) yields the inequality in (32), i.e.

$$
2 \int_{\underline{x}}^{\bar{x}} \Phi(s)[1-\Phi(s)] d s \leq \omega .
$$

\footnotetext{
${ }^{21}$ Again, ties have probability zero and can be broken in any way.
} 
We thus conclude that (32) is necessary for the implementability of $p^{*}$.

We now come to the sufficiency part. Our task is to show that whenever (32) is satisfied there is an incentive compatible, individually rational, budget balanced mechanism implementing the efficient outcome. Consider the mechanism $\left(p^{*}, s^{A}, t^{A}\right)$, where the transfer rule $t^{A}$ is defined by

$$
\begin{gathered}
t_{i}^{A}\left(x_{i}, x_{-i}\right) \equiv\left\{\begin{array}{cc}
-m\left(x_{-i}\right)-\frac{\omega}{2}, & \text { if } x_{i}>x_{-i}, \\
m\left(x_{i}\right)+\frac{\omega}{2}, & \text { if } x_{i}<x_{-i},
\end{array}\right. \\
m(t) \equiv \frac{\int_{t}^{\bar{x}}(1-\Phi(s))^{2} d s}{(1-\Phi(t))^{2}}+t .
\end{gathered}
$$

Notice that $m(t)$ is increasing, since

$$
m^{\prime}(t)=\frac{2\left(\int_{t}^{\bar{x}}(1-\Phi(s))^{2} d s\right)}{(1-\Phi(t))^{2}} \mu(t)
$$

where $\mu(t)=\frac{\phi(t)}{1-\Phi(t)}$. Notice further that

$$
\lim _{t \rightarrow \bar{x}} m(t)=\bar{x}
$$

We now show that, if (32) holds, the pair $\left(s^{A}, t^{A}\right)$ implements $p^{*}$. First, it is straightforward to check that $t_{0}^{A}\left(x_{0}, x_{1}\right)+t_{1}^{A}\left(x_{1}, x_{0}\right)=0$ for each pair $\left(x_{0}, x_{1}\right)$, thus ex post budget balance holds. Individual rationality is satisfied for all types if it is satisfied for type $\bar{x}$. Since we have

$$
\begin{gathered}
U(\bar{x})=\int_{\underline{x}}^{\bar{x}}\left(\omega+\bar{x}+x-m(x)-\frac{\omega}{2}\right) d \Phi(x)-\bar{x} \\
=\frac{\omega}{2}-\int_{\underline{x}}^{\bar{x}}\left(\frac{\int_{x}^{\bar{x}}(1-\Phi(s))^{2} d s}{(1-\Phi(x))^{2}}\right) d \Phi(x) \\
=\frac{\omega}{2}-\int_{\underline{x}}^{\bar{x}} \Phi(s)(1-\Phi(s)) d s
\end{gathered}
$$

our assumption implies $U(\bar{x}) \geq 0$. 
To check incentive compatibility observe that the interim utility of a type $x_{i}$ announcing $\widehat{x}_{i}$ is

$$
\begin{aligned}
\widehat{U}_{i}\left(\widehat{x}_{i} ; x_{i}\right) & =\int_{\underline{x}_{i}}^{\widehat{x}_{i}}\left[\omega+x_{-i}+x_{i}-m\left(x_{-i}\right)-\frac{\omega}{2}\right] d \Phi\left(x_{-i}\right)+\int_{\widehat{x}_{i}}^{\bar{x}_{i}}\left(m\left(\widehat{x}_{i}\right)+\frac{\omega}{2}\right) d \Phi\left(x_{-i}\right)-x_{i} \\
& =\frac{\omega}{2}+\int_{\underline{x}_{i}}^{\widehat{x}_{i}}\left(x_{-i}-m\left(x_{-i}\right)\right) d \Phi\left(x_{-i}\right)+\left(m\left(\widehat{x}_{i}\right)-x_{i}\right)\left[1-\Phi\left(\widehat{x}_{i}\right)\right] .
\end{aligned}
$$

Using (47) and (48), the first derivative becomes

$$
\begin{aligned}
\frac{\partial \widehat{U}_{i}\left(\widehat{x}_{i}, x_{i}\right)}{\partial \widehat{x}_{i}} & =\left(\widehat{x}_{i}+x_{i}-2 m\left(\widehat{x}_{i}\right)\right) \phi\left(\widehat{x}_{i}\right)+m^{\prime}\left(\widehat{x}_{i}\right)\left[1-\Phi\left(\widehat{x}_{i}\right)\right] \\
& =\left(x_{i}-\widehat{x}_{i}\right) \phi\left(\widehat{x}_{i}\right),
\end{aligned}
$$

which is positive for $\widehat{x}_{i}<x_{i}$ and negative for $\widehat{x}_{i}>x_{i}$. This concludes the proof.

Proof of Proposition 4. The interim expected surplus function for agent $i$ when $x_{i}^{\prime}$ is announced, $x_{i}$ is the true value and the other agent is telling the truth is

$$
\begin{aligned}
\widehat{U}_{i}\left(x_{i}^{\prime}, x_{i}\right)= & E_{x_{j}}\left[p\left(x_{i}^{\prime}, x_{j}\right) s_{0 i}^{1}\left(x_{i}^{\prime}, x_{j}\right)\left(x_{i}+x_{j}+\omega\right)\right] \\
& +E_{x_{j}}\left\{\left[1-p\left(x_{i}^{\prime}, x_{j}\right)\right] s_{i i}^{0}\left(x_{i}^{\prime}, x_{j}\right)\right\} x_{i}+E_{x_{j}}\left\{\left[1-p\left(x_{i}^{\prime}, x_{j}\right)\right] s_{j i}^{0}\left(x_{i}^{\prime}, x_{j}\right) x_{j}\right\} \\
& +T_{i}\left(x_{i}^{\prime}\right)-x_{i}, \quad i, j=0,1, \quad i \neq j ;
\end{aligned}
$$

where

$$
T_{i}\left(x_{i}^{\prime}\right) \equiv E_{x_{j}}\left[p\left(x_{i}^{\prime}, x_{j}\right) t_{i}^{1}\left(x_{i}^{\prime}, x_{j}\right)+\left(1-p\left(x_{i}^{\prime}, x_{j}\right)\right) t_{i}^{1}\left(x_{i}^{\prime}, x_{j}\right)\right] .
$$

The standard characterization of incentive compatibility implies

$$
U_{i}^{\prime}\left(x_{i}\right)=-1+E_{x_{j}}\left[p\left(x_{i}, x_{j}\right) s_{0 i}^{1}\left(x_{i}, x_{j}\right)+\left(1-p\left(x_{i}, x_{j}\right)\right) s_{i i}^{0}\left(x_{i}, x_{j}\right)\right] .
$$

Notice that, since $p\left(x_{i}, x_{j}\right) s_{0 i}^{1}\left(x_{i}, x_{j}\right)+\left[1-p\left(x_{i}, x_{j}\right)\right] s_{i i}^{0}\left(x_{i}, x_{j}\right) \leq 1$, we have $U_{i}^{\prime}\left(x_{i}\right) \leq 0$. 
Suppose first that the joint surplus

$$
\sum_{i=0}^{1} E\left[U_{i}\left(x_{i}\right)\right]=\omega E\left[p\left(x_{0}, x_{1}\right)\right]
$$

is maximized by a mechanism such that $s_{i i}^{0}\left(x_{i}, x_{j}\right)<1$. A straightforward adaptation of the argument in the proof of Lemma 3 implies that we can construct a mechanism with $s_{i i}^{0}\left(x_{i}, x_{j}\right)=1$ which still achieves the same joint surplus. The simple idea is that setting $s_{i i}^{0}\left(x_{i}, x_{j}\right)=1$ minimizes the information rents.

Therefore, we can limit attention to mechanisms that satisfy the LU property $s_{i i}^{0}\left(x_{i}, x_{j}\right) \equiv 1$ for each pair $\left(x_{i}, x_{j}\right)$. This implies

$$
U_{i}^{\prime}\left(x_{i}\right)=-E_{x_{j}}\left[p\left(x_{i}, x_{j}\right)\left(1-s_{0 i}^{1}\left(x_{i}, x_{j}\right)\right)\right]
$$

hence

$$
\begin{aligned}
& \sum_{i=0}^{1} E\left[U_{i}\left(x_{i}\right)\right]=\sum_{i=0}^{1} U_{i}(\bar{x})-\sum_{i=0}^{1} \int_{\underline{x}}^{\bar{x}} \Phi\left(x_{i}\right) U_{i}^{\prime}\left(x_{i}\right) d x_{i} \\
= & \sum_{i=0}^{1} U_{i}(\bar{x})+\int_{\underline{x}}^{\bar{x}} \sum_{i=0}^{1} \Phi\left(x_{i}\right) E_{x_{j}}\left[p\left(x_{i}, x_{j}\right)\left(1-s_{0 i}^{1}\left(x_{i}, x_{j}\right)\right)\right] d x_{i} \\
= & \sum_{i=0}^{1} U_{i}(\bar{x})+\int_{\underline{x}}^{\bar{x}} \int_{\underline{x}}^{\bar{x}}\left[\frac{\Phi\left(x_{0}\right)}{\phi\left(x_{0}\right)}\left(1-s_{00}^{1}\left(x_{0}, x_{1}\right)\right)+\frac{\Phi\left(x_{1}\right)}{\phi\left(x_{1}\right)}\left(1-s_{01}^{1}\left(x_{1}, x_{0}\right)\right)\right] p\left(x_{i}, x_{j}\right) d \Phi\left(x_{0}\right) d \Phi\left(x_{1}\right)
\end{aligned}
$$

Using the equality in (50), solving for $\sum_{i=0}^{1} U_{i}(\bar{x})$, and invoking individual rationality yields

$$
\int_{\underline{x}}^{\bar{x}} \int_{\underline{x}}^{\bar{x}}\left[\omega-\frac{\Phi\left(x_{0}\right)}{\phi\left(x_{0}\right)}\left(1-s_{00}^{1}\left(x_{0}, x_{1}\right)\right)-\frac{\Phi\left(x_{1}\right)}{\phi\left(x_{1}\right)}\left(1-s_{01}^{1}\left(x_{1}, x_{0}\right)\right)\right] p\left(x_{i}, x_{j}\right) d \Phi\left(x_{0}\right) d \Phi\left(x_{1}\right) \geq 0 .
$$

The optimization program can now be rewritten as maximizing the joint surplus, subject to (51). To see that the acquisition sharing rule is optimal, note that the constraint in (51) must be binding and, if $\frac{\Phi(\cdot)}{\phi(\cdot)}$ is nondecreasing, replacing any $s$ with $s^{A}$ creates slack. Finally, the payment function can be constructed as in the proof of Proposition 3. 


\section{References}

[1] Andrade, G. and E. Stafford, 'Investigating the Economic Role of Mergers', The Journal of Corporate Finance, 10 (2004), 1-36.

[2] Boone, A. and H. Mulherin, 'How Are Firms Sold?', The Journal of Finance, 2005, forthcoming.

[3] Che, Y.-K. and T. Lewis, 'The Role of Lockups in Takeover Contests', mimeo, Duke University (2003) http://faculty.fuqua.duke.edu/bio/lewis/RANDLockups.pdf

[4] Crèmer J., 'Auctions with Contingent Payments: Comment', American Economic Review, 77 (1987), 746 .

[5] Crèmer J. and R. McLean, 'Full Extraction of the Surplus in Bayesian and Dominant Strategy Auctions', Econometrica 56, (1988) 1247-57.

[6] Datar, S., R. Frankel, and M. Wolfson, 'Earnouts: The Effects of Adverse Selection and Agency Costs on Acquisition Techniques', The Journal of Law, Economics, and Organization 17 (2001), 201-237.

[7] DeMarzo P., I. Kremer and A. Skrzypacz, 'Bidding with Securities: Auctions and Security Design', American Economic Review, (2005) forthcoming.

[8] Eckbo, B.E., R. Giammarino and R. Heinkel, 'Asymmetric Information and the Medium of Exchange in Takeovers: Theory and Tests', Review of Financial Studies, 3 (1990), 651-675.

[9] Fieseler K., T. Kittsteiner and B. Moldovanu, 'Partnerships, Lemons and Efficient Trade', Journal of Economic Theory 113 (2003), 223-234.

[10] Gilson, R. J. and B. S. Black, The Law and Finance of Corporate Acquisitions, 2d ed., (Westbury, NY: The Foundation Press, Inc., 1995) 
[11] Gort, M., 'An Economic Disturbance Theory of Mergers', Quarterly Journal of Economics, 83 (1969), 624-642.

[12] Gorton, G, M. Kahl, and R. Rosen, 'Eat Or Be Eaten: A Theory Of Mergers and Merger Waves', (2000) Unpublished working paper, The Wharton School.

[13] Hansen R.G., 'Auctions with Contingent Payments', American Economic Review, 75 (1985), $862-5$.

[14] Harford, J. 'What Drives Merger Waves?', The Journal of Financial Economics, 77(3) (2005), 529-560.

[15] Hietala, P., S. Kaplan and D.T. Robinson, 'What is the Price of Hubris? Using Takeover Battles to Infer Overpayments and Synergies', Financial Management, 32 (2003), 1-32.

[16] Jovanovic, B., and P. Rousseau, 'The Q-Theory of Mergers', The American Economic Review, (Papers and Proceedings), 92(2) (2002), 198-204.

[17] Jehiel P. and B. Moldovanu, 'Efficient Design with Interdependent Values', Econometrica, 69 (2001), 1237-59.

[18] Krishna V., Auction Theory, Academic Press, San Diego, USA (2002)

[19] McAfee, R. P., 'Endogenous Availability, Cartels, and Merger in an Equilibrium Price Dispersion', Journal of Economic Theory, 62 (1994), 24-47.

[20] McAfee, R. P. and P. Reny, 'Correlated Information and Mechanism Design', Econometrica 60 (1992), 395-421.

[21] 'Mergerstat Review' (2004) www.mergerstat.com.

[22] Maksimovic, V., and G. Phillips, 'The Market for Corporate Assets: Who Engages in Mergers and Asset Sales and Are There Efficiency Gains?', Journal of Finance, 56 (2001), 2019-2065. 
[23] Makowski, L. and C. Mezzetti,'The Possibility of Efficient Mechanisms for Trading an Indivisible Object,' The Journal of Economic Theory 59 (1993), 451-465

[24] Mezzetti C., 'Mechanism Design with Interdependent Valuations: Efficiency' Econometrica, $72(2002), 1617-1626$.

[25] Mitchell, M.L., and J.H. Mulherin, 'The Impact of Industry Shocks on Takeover and Restructuring Activity', The Journal of Financial Economics, 41(1996), 193-229.

[26] Mulherin, J.H., and A.L. Boone, 'Comparing Acquisitions and Divestitures', The Journal of Corporate Finance, 6(2), (2000), 117-139.

[27] Myers, Stewart and Nicholas Majluf, 'Corporate Financing and Investment Decisions when Firms Have Information That Investors Do Not Have', Journal of Financial Economics, 13 (1984), 187-221.

[28] Myerson, R. and M. Satterthwaite, 'Efficient Mechanisms for Bilateral Trading', Journal of Economic Theory 28 (1983), 265-281.

[29] Officer, Micah, "Collars in Merger Negotations," Journal of Finance, 59 (2004), 2719-2743.

[30] Rhodes-Kropf, M. and D.T. Robinson, 'The Market for Mergers and the Boundaries of the Firm,' working paper, Duke University and Columbia University, 2005.

[31] Rhodes-Kropf, M. and S. Viswanathan, 'Corporate Reorganizations and Non-Cash Auctions', Journal of Finance, 55 (2000), 1807-1854.

[32] Rhodes-Kropf, M. and S. Viswanathan, 'Market Valuation and Merger Waves', Journal of Finance, 59 (2004), 2685-2718.

[33] Rhodes-Kropf, M., D.T. Robinson and S. Viswanathan, 'Valuation Waves and Merger Waves: The Empirical Evidence', Journal of Financial Economics, 77 (2005), 561-603. 
[34] Roll, R., 'The Hubris Hypothesis of Corporate Takeovers', The Journal of Business, 59(2) (1986), 197-216.

[35] Samuelson W., 'Auctions with Contingent Payments: Comment', American Economic Review, 77 (1987), 740-5.

[36] Shleifer, Andrei and Robert Vishny, 'Stock Market Driven Acquisitions', Journal of Financial Economics, 72 (2003), 295-311.

[37] Weston, J. F., M. L. Mitchell, and J. H. Mulherin, Takeovers, Restructuring and Corporate Governance, 4th ed., Upper Saddle River, NJ: Pearson Prentice Hall, Inc. 2003.

[38] Williams S., 'A Characterization of Efficient, Bayesian Incentive Compatible Mechanisms', Economic Theory, 14 (1999), 155-180. 